\title{
Designing Robot Behavior in Human-Robot Interactions
}

\section{Changliu Liu}

Robotics Institute, Carnegie Mellon University

Pittsburgh, Pennsylvania, USA

\section{Te Tang}

Advanced Research Laboratory

FANUC America Corporation, Union City, California, USA

\section{Hsien-Chung Lin}

Advanced Research Laboratory

FANUC America Corporation, Union City, California, USA

\section{Masayoshi Tomizuka}

Department of Mechanical Engineering

University of California, Berkeley, California, USA

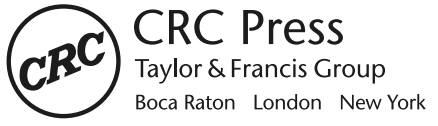

CRC Press is an imprint of the 
CRC Press

Taylor \& Francis Group

6000 Broken Sound Parkway NW, Suite 300

Boca Raton, FL 33487-2742

(C) 2019 by Taylor \& Francis Group, LLC

CRC Press is an imprint of Taylor \& Francis Group, an Informa business

No claim to original U.S. Government works

\section{Version Date: 20190712}

International Standard Book Number-13: 978-0-367-17969-4 (Hardback)

Th is book contains information obtained from authentic and highly regarded sources. Reasonable eff orts have been made to publish reliable data and information, but the author and publisher cannot assume responsibility for the validity of all materials or the consequences of their use. Th e authors and publishers have attempted to trace the copyright holders of all material reproduced in this publication and apologize to copyright holders if permission to publish in this form has not been obtained. If any copyright material has not been acknowledged please write and let us know so we may rectify in any future reprint.

Except as permitted under U.S. Copyright Law, no part of this book may be reprinted, reproduced, transmitted, or utilized in any form by any electronic, mechanical, or other means, now known or hereafter invented, including photocopying, microfi lming, and recording, or in any information storage or retrieval system, without written permission from the publishers.

For permission to photocopy or use material electronically from this work, please access www.copyright.com (http://www.copyright.com/) or contact the Copyright Clearance Center, Inc. (CCC), 222 Rosewood Drive, Danvers, MA 01923, 978-750-8400. CCC is a not-for-profi t organization that provides licenses and registration for a variety of users. For organizations that have been granted a photocopy license by the CCC, a separate system of payment has been arranged.

Trademark Notice: Product or corporate names may be trademarks or registered trademarks, and are used only for identification and explanation without intent to infringe.

\begin{tabular}{l} 
Library of Congress Cataloging-in-Publication Data \\
\hline Names: Liu, Changliu, 1990- author. I Tang, Te, author. I Lin, Hsien-Chung, \\
author. I Tomizuka, M., author. \\
Title: Designing robot behavior in human-robot interactions / Changliu Liu, \\
Robotic Institute, Carnegie Mellon University, Pittsburgh, Pennsylvania, \\
USA, Te Tang, Department of Mechanical Engineering, University of \\
California, Berkeley, USA, Hsien-Chung Lin, Research Engineer at FANUC \\
America Corp, San Francisco, California, USA, Masayoshi Tomizuka, \\
Department of Mechanical Engineering, University of California, \\
Berkeley, USA. \\
Description: Boca Raton : CRC Press, Taylor \& Francis Group, [2019] | "A \\
science publishers book." I Includes bibliographical references and \\
index. I Summary: "In this book, we have set up a unified analytical \\
framework for various human-robot systems, which involve peer-peer \\
interactions (either space-sharing or time-sharing) or hierarchical \\
interactions. A methodology in designing the robot behavior through \\
control, planning, decision and learning is proposed. In particular, the \\
following topics are discussed in-depth: safety in the process of during \\
human-robot interactions, efficiency in real-time robot motion planning, \\
imitation of human behaviors from demonstration, dexterity of robots to \\
adapt to different environments and tasks, cooperation among robots and \\
humans with conflict resolution. These methods are applied in various \\
scenarios, such as human-robot collaborative assembly, robot skill \\
learning from human demonstration, interaction between autonomous and \\
human-driven vehicles, etc"-- Provided by publisher. \\
Identifiers: LCCN 2019026334 | ISBN 9780367179694 (hardcover : acid-free \\
paper) \\
Subjects: LCSH: Human-robot interaction. I Robotics. \\
Classification: LCC TJ211.49.L58 2019 I DDC 629.8/924019---dc23 \\
LC record available at https://lccn.loc.gov/2019026334 \\
\hline
\end{tabular}

\section{Visit the Taylor \& Francis Web site at http://www.taylorandfrancis.com}




\section{Preface}

Human-robot interactions (HRI) have been recognized to be a key element of future robots in many application domains such as manufacturing and transportation, which have huge social and economic impacts. Future robots are envisioned to be acting like humans, which are independent entities that make decisions for themselves; intelligent actuators that interact with the physical world; and involved observers that have rich senses and critical judgements. Most importantly, they are entitled social attributions to build relationships with humans. We call them co-robots.

Technically, it is challenging to design the behavior of co-robots. Unlike traditional robots which work in structured and deterministic environments, co-robots need to operate in highly unstructured and stochastic environments. The book is dedicated to study the methodologies to ensure that co-robots operate efficiently and safely in dynamic uncertain environments.

This book sets up a unified analytical framework for various human-robot systems, which involve peer-peer interactions or hierarchical interactions. Various methods to design robot behavior through control, planning, decision, and learning are proposed. In particular, the following topics are discussed: safety during human robot interactions, efficiency in real-time robot motion planning, imitation of human behaviors by robot, dexterity of robots to adapt to different environments and tasks, cooperation among robots and humans and conflict resolution. The proposed methods have been applied on various scenarios, such as human-robot collaborative assembly, robot skill learning from human demonstration, interaction between autonomous and human-driven vehicles, etc. 


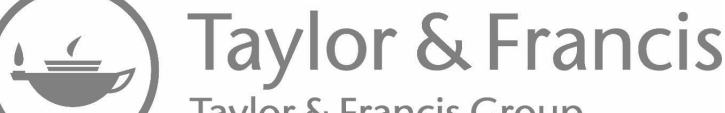

Taylor \& Francis Group

http://taylorandfrancis.com 


\section{Acknowledgement}

This book is based on the doctoral researches of Prof. Liu, Dr. Tang, and Dr. Lin in Prof. Tomizuka's group at University of California, Berkeley. A very sincere gratitude goes out to all the collaborators on various topics:

1. Dr. Wei Zhan on speed profile planning in Chapter 4;

2. Dr. Yu Zhao and Dr. Wenjie Chen on force control by imitation learning in Chapter 5;

3. Mr. Changhao Wang on the framework of analogy learning in Chapter 6;

4. Prof. Wenlong Zhang on Blame-All strategy in Chapter 7;

5. Prof. Chung-Wei Lin and Dr. Shinichi Shiraishi on distribution conflict resolution in Chapter 7;

6. Mr. Jianyu Chen and Dr. Trong-Duy Nguyen on ROAD in Chapter 8;

7. Dr. Yongxiang Fan on grasp planning by analogy learning in Chapter 9;

8. Ms. Yujiao Cheng on SERoCS in Chapter 10.

The authors are very grateful to all the sponsors of the works presented in this book, including the National Science Foundation, and industrial sponsors: FANUC Corporation, Denso International America, and Toyota InfoTechnology Center. Their support and insightful discussions have enriched the results of this book.

Finally, the authors would like to thank many of their colleagues, students, and friends for their precious suggestions. In particular, we would like to thank Prof. Xu Chen, Mr. Weiye Zhao, Mr. Jaskaran Grover, and Mr. Angelos Mavrogiannis. 


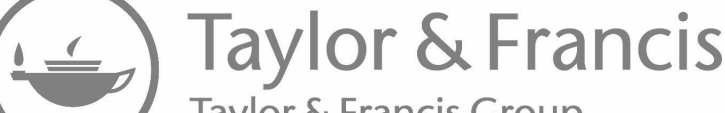

Taylor \& Francis Group

http://taylorandfrancis.com 


\section{Contents}

Preface

iii

Acknowledgement v

Nomenclature xiii

\section{PART I INTRODUCTION}

1. Introduction 3

1.1 Human-Robot Interactions: An Overview 3

1.2 Modes of Interactions 4

1.3 Robot Behavior System 7

1.3.1 Overview

1.3.2 Knowledge 11

$\begin{array}{lll}1.3 .3 & \text { Logic } & 14\end{array}$

1.3.4 Learning Process 16

1.4 Design Objectives 17

$\begin{array}{lll}1.4 .1 \text { Safety } & 17\end{array}$

$\begin{array}{lll}1.4 .2 & \text { Efficiency } & 18\end{array}$

$\begin{array}{lll}1.4 .3 & \text { Imitation } & 19\end{array}$

1.4.4 Dexterity 20

1.4.5 Cooperation 20

1.5 System Evaluation 21

1.5.1 Theoretical Evaluation $\quad 21$

1.5.2 Experimental Evaluation $\quad 22$

1.6 Outline of the Book 24

2. Framework 27

2.1 Multi-Agent Framework 27

2.1.1 The General Multi-Agent Model 27

2.1.2 Models for Different Modes of Interactions 28

2.1.3 Features of Human-Robot Systems 30

2.2 Agent Behavior Design and Architecture 31

2.2.1 Model-Based Design 31

2.2.2 Model-Free Design 37

2.3 Conclusion 41 


\section{PART II THEORY}

3. Safety during Human-Robot Interactions 45

3.1 Overview 45

3.2 Safety-Oriented Behavior Design $\quad 46$

$\begin{array}{lll}3.3 & \text { Safe Set Algorithm } & 49\end{array}$

3.3.1 The Algorithm 49

3.3.2 Example: Planar Robot Arm $\quad 50$

3.3.3 Example: Vehicle $\quad 53$

$\begin{array}{ll}3.4 & \text { Safe Exploration Algorithm }\end{array}$

3.4.1 The Algorithm $\quad 54$

3.4.2 Example: Local Planning of a Vehicle 56

3.5 An Integrated Method for Safe Motion Control 59

$\begin{array}{ll}3.6 \text { Conclusion } & 59\end{array}$

4. Efficiency in Real-Time Motion Planning 61

$\begin{array}{lll}4.1 & \text { Overview } & 61\end{array}$

4.2 Problem Formulation $\quad 62$

4.3 Optimization-Based Trajectory Planning 64

4.3.1 Problem Formulation $\quad 65$

4.3.2 Quadratic Approximation 66

4.3.3 Examples: Trajectory Planning for Various Systems 68

4.4 Optimization-Based Speed Profile Planning 71

$\begin{array}{lll}\text { 4.4.1 Problem Formulation } & 72\end{array}$

4.4.2 Quadratic Approximation $\quad 75$

4.4.3 Example: Vehicle Speed Profile Planning 77

4.5 Optimization-Based Layered Planning 78

$\begin{array}{lll}4.6 \text { Conclusion } & 81\end{array}$

5. Imitation: Mimicking Human Behavior 83

$\begin{array}{llr}5.1 & \text { Overview } & 83\end{array}$

$\begin{array}{lll}5.2 & \text { Imitation for Prediction } & 83\end{array}$

5.2.1 Classification of Behaviors $\quad 84$

5.2.2 Adaptation of Behavior Models 86

$\begin{array}{lll}5.3 & \text { Imitation for Action } & 89\end{array}$

5.3.1 Imitation Learning by Gaussian Mixture Model 90

5.3.2 Interpreting GMR from Mechanics, Point of View 92

5.3.3 Stability Condition of the Closed-Loop System 93

$\begin{array}{lll}5.4 \text { Conclusion } & 96\end{array}$ 
6. Dexterity: Analogy Learning to Expand Robot Skill Sets 97

6.1 Overview 97

6.2 Concept of Analogy Learning 97

6.3 Advantages of Analogy Learning 98

6.3.1 Learn from SMALL Data 99

6.3.2 Robustness to Input Noise 100

6.3.3 Guaranteed Collision-Free Property 101

6.4 Structure Preserved Registration for Analogy Learning 103

6.4.1 Node Registration with Gaussian Mixture Model 103

6.4.2 Regularization on Local Structure 105

6.4.3 Regularization on Global Topology 108

6.4.4 Closed-form Solution for Transformation Function 108

6.4.5 Implementation Acceleration 113

6.5 Experimental Study 113

6.6 Conclusion 117

7. Cooperation: Conflict Resolution during Interactions 119

$\begin{array}{lll}7.1 & \text { Overview } & 119\end{array}$

7.2 Dynamics of Multi-Agent Systems 121

7.2.1 Simultaneous Dynamic Game 121

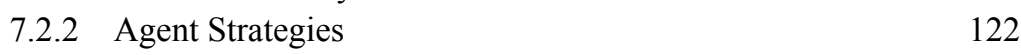

7.2.3 Multi-Agent Learning 123

7.2.4 Trapped Equilibrium 124

7.3 Cooperation under Information Asymmetry 125

$\begin{array}{ll}\text { 7.3.1 Quadratic Game and Nash Equilibrium } & 125\end{array}$

$\begin{array}{lll}\text { 7.3.2 } & \text { Blame-Me Strategy } & 128\end{array}$

$\begin{array}{lll}\text { 7.3.3 Blame-All Strategy } & 129\end{array}$

7.3.4 Example: Robot-Robot Cooperation 131

7.4 Conflict Resolution through Communication 133

7.4.1 Conflict Resolution and Nash Equilibria 134

7.4.2 Communication Protocol and Strategy 136

7.4.3 Example: Unmanaged Intersections 144

7.5 Conclusion 146

\section{PART III APPLICATIONS}

8. Human-Robot Co-existence: Space-Sharing Interactions 149

8.1 Overview 149

8.2 Robot Safe Interaction System for Industrial Robots $\quad 150$

8.2.1 The Optimization Problem 151

8.2.2 The Robot Safe Interaction System 152 
8.2.3 Case Studies 156

8.2.4 Discussion and Conclusion 159

8.3 Robustly-Safe Automated Driving System 159

8.3.1 Multi-Agent Traffic Model 160

8.3.2 Prediction and Planning in the ROAD System 162

$\begin{array}{lll}\text { 8.3.3 Case Studies } & 168\end{array}$

8.3.4 Discussion and Conclusion $\quad 172$

8.4 Conclusion 174

9. Robot Learning from Human: Hierarchical Interactions 175

9.1 Overview 175

9.2 Remote Lead Through Teaching for Implementing 175 Imitation Learning

9.2.1 Human Demonstration Phase 175

9.2.2 Skill Learning Process 176

9.2.3 Robot Reproduction Phase 177

9.2.4 Experimental Study 177

9.3 Robotic Grasping by Analogy Learning 180

9.3.1 Background of Robotic Grasping 181

9.3.2 Grasp Planning with Human Demonstration 182

9.3.3 Grasping Pose Transferring by Analogy Learning 183

$\begin{array}{ll}\text { 9.3.4 Experimental Study } & 187\end{array}$

9.4 Robotic Motion Re-Planning by Analogy Learning 190

9.4.1 Path Re-Planning by Analogy Learning 192

9.4.2 Trajectory Re-Planning by Analogy Learning 195

9.5 Conclusion 198

10. Human-Robot Collaboration: Time-Sharing Interactions 201

10.1 Human-Robot Collaboration in Manufacturing 201

10.2 Safe and Efficient Robot Collaborative System 202

10.2.1 Mathematical Problem 202

10.2.2 Architecture of SERoCS 203

10.2.3 Example 204

10.3 Experimental Study 204

10.3.1 Experiment Setup 204

10.3.2 Validation of the Environment Monitoring 206

10.3.3 Robots in the Idle Mode 207

10.3.4 Human-Robot Collaborative Assembly 209

10.4 Conclusion 213 


\section{PART IV Conclusion}

11. Vision for Future Robotics and Human-Robot Interactions 217

$\begin{array}{ll}11.1 \text { Roadmap for the Future } & 217\end{array}$

$\begin{array}{ll}\text { 11.1.1 The Demand Side } & 217\end{array}$

$\begin{array}{ll}\text { 11.1.2 Future Robotics } & 218\end{array}$

11.1.3 Human-Robot Relationships $\quad 219$

$\begin{array}{ll}\text { 11.1.4 Ethical Issues } & 219\end{array}$

11.2 Conclusion of the Book 220

$\begin{array}{ll}\text { References } & 221\end{array}$

$\begin{array}{ll}\text { Index } & \mathbf{2 3 5}\end{array}$

$\begin{array}{lr}\text { Color Section } & \mathbf{2 3 7}\end{array}$ 


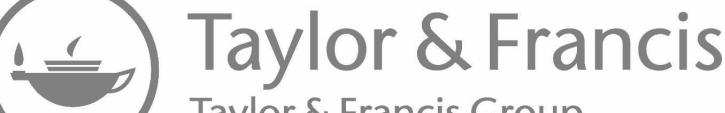

Taylor \& Francis Group

http://taylorandfrancis.com 


\section{Nomenclature}

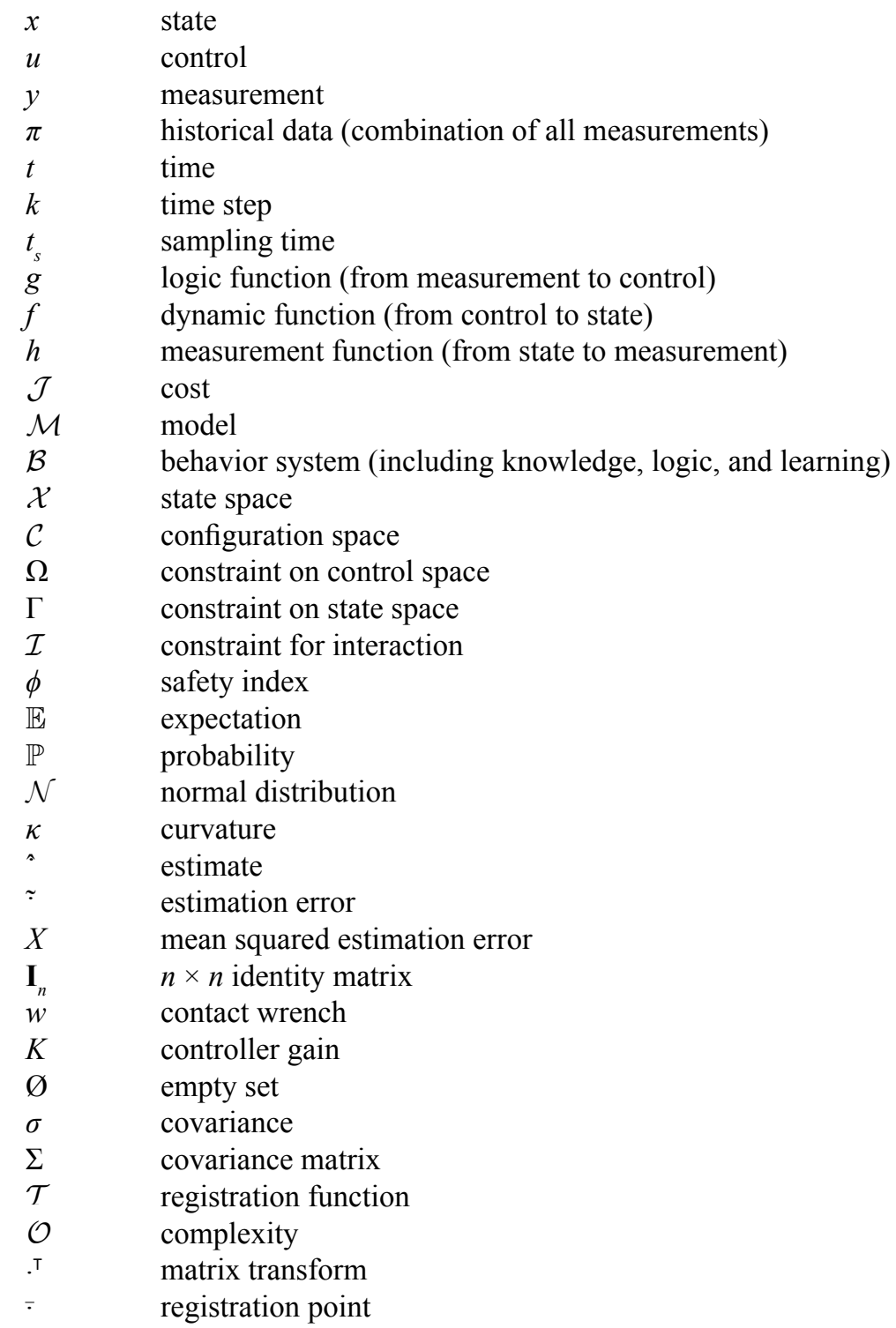




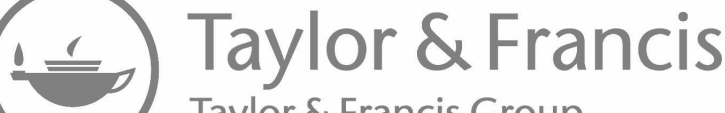

Taylor \& Francis Group

http://taylorandfrancis.com 
Part I

Introduction 


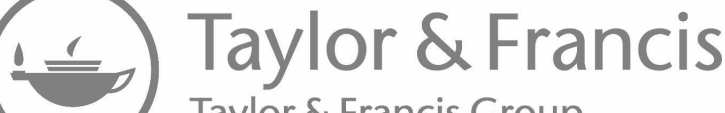

Taylor \& Francis Group

http://taylorandfrancis.com 


\section{Introduction}

\subsection{HUMAN-ROBOT INTERACTIONS: AN OVERVIEW}

Human-robot interactions (HRI) have been recognized to be a key element of future robots in many application domains such as manufacturing, transportation, service, and entertainment. In factories, robots are expected to collaborate with human workers [109]. Manufacturers are interested in combining human's flexibility and robot's productivity in flexible production lines [102]. In transportation systems, autonomous vehicles are expected to interact with various road participants, including human-driven vehicles and pedestrians. In hospitals, medical robots are expected to help doctors to perform surgeries on patients. Exoskeleton robots are expected to assist stroke patients to walk [100]. Nursing robots [169] are expected to take care of patients. Robot guide dogs [203] are expected to help blind people.

These applications entail huge social and economic impacts [52, 34]. Future robots are envisioned to be acting like humans, which are independent entities that make decisions for themselves; intelligent actuators that interact with the physical world; and involved observers that have rich senses and critical judgements. Most importantly, they are entitled social attributions to build relationships with humans [63]. We call these robots co-robots.

Technically, it is a challenge to design the behavior of co-robots. Unlike conventional robots that work in structured and deterministic environments, co-robots need to operate in highly unstructured and stochastic environments. The skill sets that have been developed for conventional robots can no longer meet the needs of corobots. This book explores methodologies to equip co-robots with desired skill sets, in order to enable wide adoption of co-robots in various interactive environments, as mentioned earlier. The fundamental research question to address in this book is how to ensure that co-robots operate efficiently and safely in dynamic uncertain environments. Regarding broadness and complexity of HRI [52], the following four aspects of the fundamental question are considered:

\section{Diverse modes of interactions}

The potential applications of co-robots lie in various domains, with different modes of interactions [167, 111, 55]. For example, humans and robots can be collaborators in factories, while automated vehicles and vehicles driven by human drivers are competitors for limited road resources on public roads. Different modes of interactions imply different system dynamics as well as diverse performance requirements for the robot. Moreover, the relationship that a robot builds with a human may be dynamic, i.e., the mode of interaction may change over time. A unified model for various HRI 
applications is needed in order to provide a comprehensive understanding of HRI, guide the design of the robot behavior, and serve as an analytical framework for performance evaluation of the human-robot systems.

\section{Design of the robot behaviors}

Behavior is the way in which one acts or conducts oneself, especially towards others. Action can be taken in multiple ways. For a humanoid robot, it can be physical movement, gesture, facial expression, language, etc. For an automated vehicle, it can be physical movements, turning on lights, honking, etc. We study the methodology of behavior design, i.e., how to realize the design goal (to ensure that co-robots operate efficiently and safely in dynamic uncertain environments) within the design scope (the inputs and outputs of the robotic system). This book mainly considers physical movements of co-robots.

\section{Software embodiment of the behavior and its computation efficiency}

The designed behavior is embodied as robot software. The complexity of the software will increase dramatically when the environment or the task becomes more complex. To ensure timely responses to environmental changes and guarantee safety during operation, real-time computation and actuation is crucial. Efficient algorithms are highly demanded.

\section{Analysis, synthesis and evaluation of complex human-robot systems}

The effectiveness of the designed robot behavior needs to be evaluated in humanrobot systems. The evaluation can be performed theoretically as well as experimentally. The challenge in theoretical analysis is the complication of interweaving software modules. The difficulty in conducting experiments is that the tolerance of failure is extremely low when human subjects are in the loop, i.e., human safety is critical. Moreover, it is difficult to repeat some human-in-the-loop experiments due to the stochasticity of human behaviors. It is important to develop effective evaluation platforms for human-robot systems.

The focus of this book is to (1) set up a unified analytical framework for various human-robot systems; (2) establish methodologies to design robot behaviors under the framework that address the fundamental question. The considerations in the above four aspects will be elaborated in the following sections.

\subsection{MODES OF INTERACTIONS}

Interaction between a human and a robot may have various modes. We divide it into two kinds of relationships: parallel relationships and hierarchical relationships. 

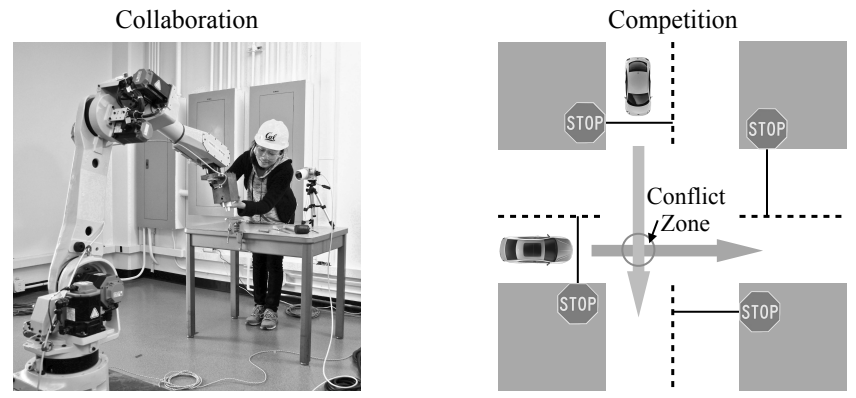

(a) Parallel relationships in human-robot interactions.

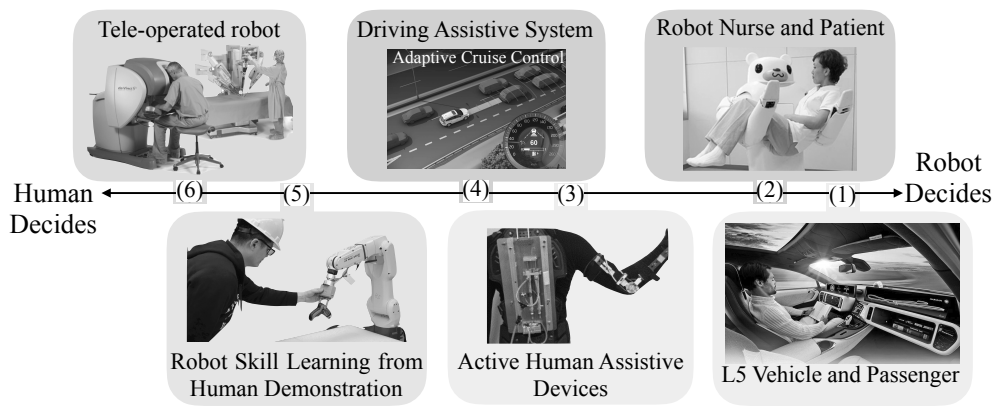

(b) Hierarchical relationships in human-robot interactions.

Figure 1.1: Various modes of human-robot interactions.

Color version at the end of the book

\section{Parallel HRI}

In a parallel relationship, a human and a robot are two independent entities who make their own decisions. In literature, parallel interaction is also called peer-peer interaction [55]. Typical examples of parallel relationships are (1) the interaction between an automated vehicle and a human-driven vehicle and (2) the interaction between an industrial co-robot and a human worker in production lines. In either case, the robot (the automated vehicle or the industrial co-robot) and the human (the human-driven vehicle or the human worker) are peers as opposed to hierarchical relationships. In the parallel relationships shown in Fig. 1.1a, the actions of the human and the robot either need to be synchronized (e.g., when the human and the robot are moving one workpiece cooperatively) or need to be asynchronized (e.g., two vehicles cannot occupy the conflict zone at the same time when they are crossing an intersection). We call the synchronized actions collaboration and the asynchronized actions competition (since there will always be a vehicle that passes the conflict zone first). Competition is the most common interaction mode. For instance, if a human and a robot are competing for space, the competition can be understood as collision avoidance. 


\section{Hierarchical HRI}

In hierarchical relationships, either the human or the robot transfers the responsibility of making decisions to the other, completely or partially. Typical examples in hierarchical relationships are listed below, which is also shown in Fig. 1.1b.

1. The interaction between an automated vehicle and a passenger inside a vehicle, where the human passenger allows the vehicle to make driving decisions. ${ }^{1}$

2. The interaction between a robot nurse and a patient, where the robot decides the moving trajectory for the patient. ${ }^{2}$

3. The interaction between a human and a human-assistive device such as an exoskeleton. The human can be guided by the robot, but can also "fight" against the robot.

4. The interaction between a human driver and a driver-assistance system. ${ }^{3}$ The driver-assistance system can serve as a guardian angel [89] that allows the driver to decide in safe situations and takes over during an emergency, or as an assistant that takes charge in safe situations and asks the human to take charge of emergencies. ${ }^{4}$

5. The interaction between a human and a robot when the human teaches the robot a skill by kinesthetic teaching. In this case, the robot is following the moving trajectory taught by the human.

6. The interaction between a human tele-operator and a tele-operated robot, where the robot purely follows the command from the human. ${ }^{5}$

As discussed in the above examples, the allocation of responsibilities varies for different hierarchical interactions. The robot purely follows the human's command in one extreme, while the human purely follows the robot's motion in another extreme. When human dominates the decision process, it is called supervisory interaction [153] in literature. Allocation of responsibility can change throughout time as discussed in the guardian angel case for driver-assistance systems.

The modes of interactions among multiple humans and robots can be built from those basic interaction modes between one human and one robot. A multi-agent framework will be proposed in Chapter 2 to provide a unified framework to analyze various kinds of interactions, where every intelligent entity (human or robot) is viewed as one agent. For simplicity, we do not distinguish between a robot and a co-robot literally in the following discussion.

\footnotetext{
${ }^{1}$ Online figure: http://www.autonews.com/article/20160111/OEM06/301119965/autonomousvehicle-architects-begin-to-contemplate-the-human-inside.

${ }^{2}$ Online figure: http://newatlas.com/riba-robot-nurse/12693/.

${ }^{3}$ Online figure: http://editorial.autoweb.com/autowebs-guide-to-adaptive-cruise-control/.

${ }^{4}$ The level of autonomy of an intelligent vehicle is divided into six categories in the SAE International's new standard J3016 [6], which ranges between the two extremes: either the human driver makes all the decisions or the automated vehicle makes all the decisions.
}

${ }^{5}$ Online figure: http://www.telepresenceoptions.com/2014/08/r/. 


\subsection{ROBOT BEHAVIOR SYSTEM}

This book focuses on behavior design of a robot from the perspective of physical movement, e.g., how to generate safe and efficient motions during interactions.

\subsubsection{OVERVIEW}

\section{Three components}

To generate desired robot behavior, we need to

1. encode correct knowledge in the robot;

2. design a correct logic to let the robot turn the knowledge into desired actions;

3. design a learning process to update the knowledge and the logic, in order to make the robot adaptable to unforeseen environments.

Knowledge, logic, and learning are the major components of a behavior system, as shown in Fig. 1.2a. In the diagram, the robot obtains measurement $\pi$ from the human-involved environment and generates its action $u$ according to the logic.

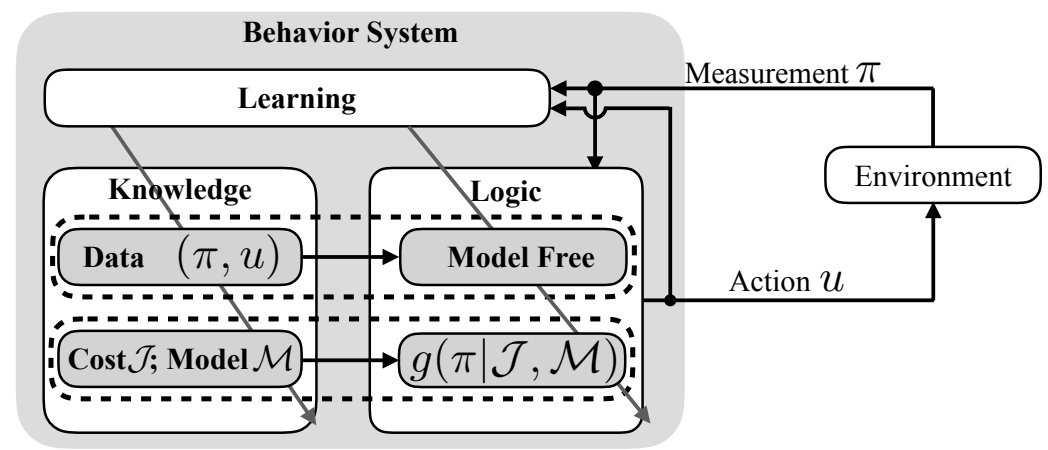

(a) Components of a behavior system.

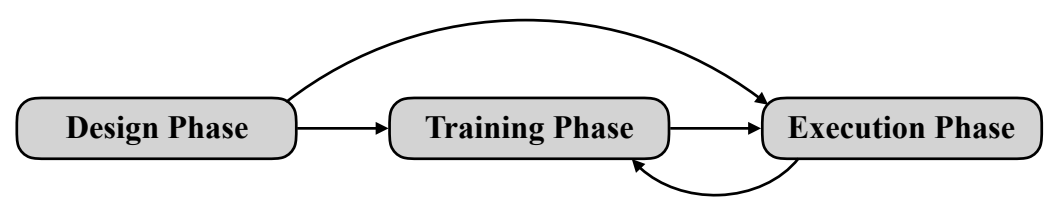

(b) The life-cycle of a behavior system.

Figure 1.2: Behavior systems.

Knowledge and logic can be considered in both the model-based framework or the model-free framework. Model-free knowledge consists of a set of data points 
$(\pi, u)$, which implicitly encodes the relationship between measurement and action. The corresponding logic is a model-free logic that acts according to the implicit mapping from $\pi$ to $u$ observed in the data points. Model-based knowledge consists of cost that describes task requirements and model that describes the dynamics of the environment. The corresponding logic $g(\pi \mid \mathcal{J}, \mathcal{M})^{6}$ is a mapping from measurement to action that depends on its knowledge: the cost $\mathcal{J}$ and the model $\mathcal{M}$.

The learning process updates the knowledge and the logic based on the data $\pi$, which is necessary since (1) the designed knowledge may not cover all possible scenarios and (2) the environment may be time varying. The mathematical formulation will be further explained in Chapter 2 .

\section{Robot lifetime}

The lifetime of a robot is divided into three phases: the design phase, the training phase and the execution phase, as shown in Fig. 1.2b. We call the first two phases offline process and the third online process. In the design phase, the human designers specify the three components in Fig. 1.2a for the robot. In the training phase, the robot can learn knowledge from experience or from human demonstration. The difference between the knowledge learned from human demonstration and the knowledge designed by human is that the former does not require the human to have a mathematical or quantitative representation of the knowledge. In many cases, such mathematical representations are hard to obtain and unintuitive for the human. For example, it is easier for a human to gesture a motion trajectory than to come up with a mathematical function of the trajectory. In the execution phase, the robot performs its task and interacts with the environment. While performing the tasks, the robot can update the knowledge or the logic through online learning. However, due to limitations in computation power, online learning is restricted to small-scale parametric adaptation. Structural changes such as learning a new skill from scratch are performed by offline learning in the training phase. The training phase and the execution phase can be performed iteratively in a lifelong learning system [212]. It is also possible that the robot goes directly from the design phase to the online execution phase without going through the training phase.

\section{Nature vs. nurture}

Knowledge is the key content of a behavioral system. How much it should be designed (nature) and how much it should be learned (nurture) still remains arguable [51].

Although knowledge can be learned, the two other components, logic and learning, are essentially algorithms to be designed. There are three ways to obtain logic $g$ as illustrated in Fig. 1.3. The contours in the figures represent the internal cost $\mathcal{J}(u, \pi)$. The darker the color, the higher the cost. Logic $g$ is a mapping from $\pi$ to $u$, and can be obtained using the following three methods. The first two methods

\footnotetext{
${ }^{6}$ The function $g$ is also called a control law in classic control theory or a control policy in decision theory.
} 


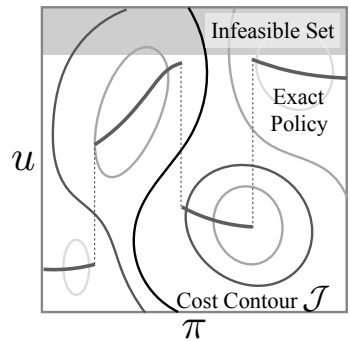

(a) Model-based explicit policy.

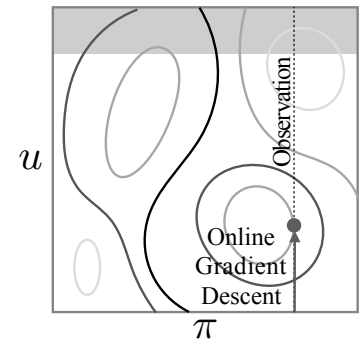

(b) Model-based implicit policy.

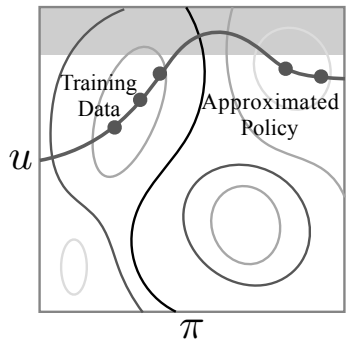

(c) Model-free policy.

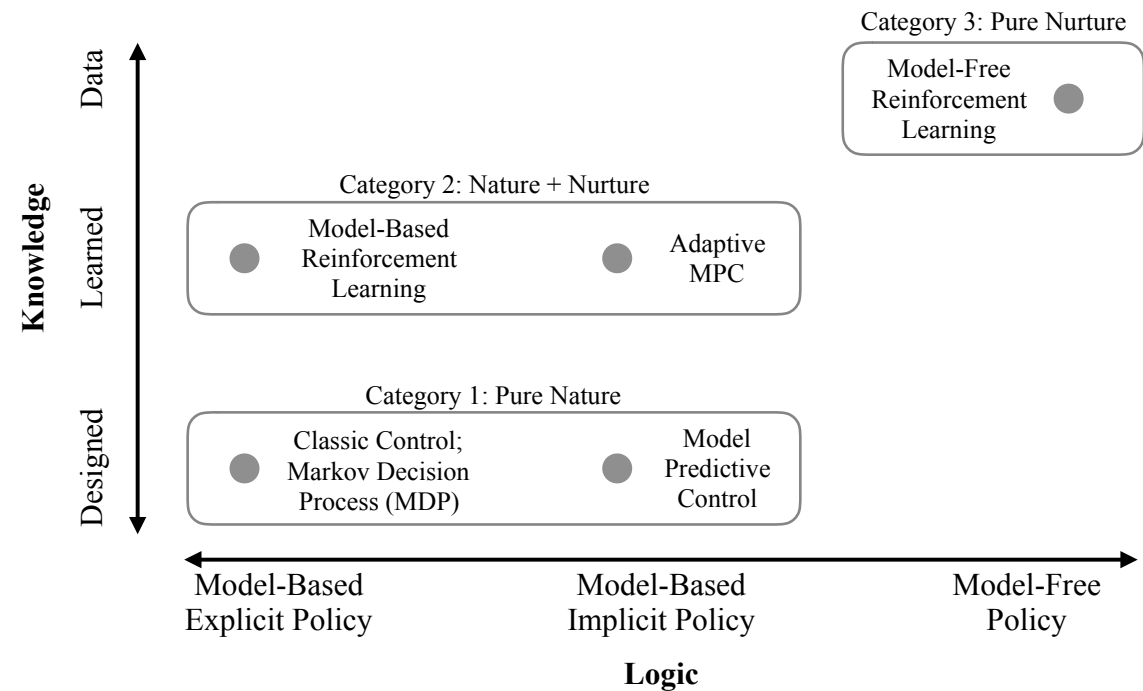

(d) Classification of behavior systems from nature to nurture.

Figure 1.3: Classification of behavior systems.

are model-based, which assume explicit $\mathcal{J}$ and $\mathcal{M}$. The last method is model-free, which only requires $(\pi, u)$ data points.

1. Model-based explicit policy.

We can explicitly solve the optimization $\min _{u} \mathcal{J}(u, \pi)$ given $\mathcal{M}$ in the design phase (offline). In this way, we obtain a model-based explicit policy $g: \pi \mapsto u$ which solves the optimization, i.e., $g(\pi):=\min _{u} \mathcal{J}(u, \pi)$, as shown in the thick curve in Fig. 1.3a. The function $g$ may be discontinuous, when the cost $\mathcal{J}$ is non-convex.

2. Model-based implicit policy.

The optimization can also be solved online in the execution phase. An algorithm (e.g., gradient descent) needs to be designed such that given any 
observation $\pi$, a desired control input $u$ can be computed. This provides a model-based implicit policy, as shown in Fig. 1.3b. When the cost $\mathcal{J}$ is non-convex, the control input $u$ computed online may only be a local optimum. The computation of $u$ is only initiated when $\pi$ is obtained in the execution phase, which is different from an explicit policy that computes $u$ for all possible $\pi$ in the design phase.

\section{Model-free policy.}

We can also approximate the policy using parametric functions (e.g., neural networks) in the training phase. Given model-free knowledge which consists of a set of data points $(\pi, u)$, the function $g$ can be approximated from the data as shown in Fig. 1.3c. Since no explicit cost or model is extracted from the data, this is a model-free logic. ${ }^{7}$

The three-component behavior system represents a variety of existing methods. We summarize the methods into the following three categories ranging from natureoriented design to nurture-oriented design, as shown in Fig. 1.3d.

\section{Category 1: Pure Nature}

The designer specifies the cost and the model and designs model-based logics to solve the optimization without any learning process. Representative methods are (1) classic control methods and Markov decision processes (MDPs) where explicit policies are obtained in the design phase (e.g., in the control of flexible robot joints [100, 214], or in safety critical situations $[80,228]$ ), and (2) model predictive control (MPC) methods where the optimization is computed in the execution phase [146, 46, 122].

\section{Category 2: Nature + Nurture}

In one case, the designer specifies the cost, designs the logic explicitly, and defers to the learning process to identify the models. Classic adaptive control and adaptive MPC belong to this category. Application of this approach in human-robot interactions can be found in [185, 131, 76, 152]. The advantage of this method is that it can account for time varying environmental changes, especially when humans are in the loop, while the designer still has control over the task performance through explicit design of the knowledge and the logic.

Another case is that the designer only designs the logic and the learning process explicitly. The knowledge is obtained in the training phase by either trial-and-error or expert demonstration. Representative methods are modelbased reinforcement learning and inverse reinforcement learning such as apprentice learning $[8,15,70]$. The learning object can either be the cost

\footnotetext{
${ }^{7}$ Though a model-free policy can well approximate the true policy in data-rich regions, it may not generalize to regions with few data. Moreover, it is hard for model-free methods to infer hard constraints from the data. In Fig. 1.3c, the model-free policy (thick curve) deviates from the exact policy in regions with few data and even violates the hard constraint (shaded infeasible set).
} 
(if the task is very complicated) or the model (if the dynamics are complicated) or both (as in cost-model coupled $Q$-learning). Application of this approach in human-robot interactions can be found in [211, 11, 162]. The advantage of this method is that mathematical modeling of the task and the environment is no longer required in the design phase.

\section{Category 3: Pure Nurture}

The designer designs the learning process explicitly, obtains model-free knowledge in the training phase, and approximates the logic using a modelfree policy, which is usually represented by a neural network. In contrast to knowledge learning in Category 2, the knowledge in this category is not explicitly learned, but implicitly encoded in the data points and in the neural network. Representative methods are model-free reinforcement learning such as deep reinforcement learning [156] and imitation learning [104]. In addition to human subjects, the object to be imitated may be a behavior system in Category 1 and Category 2 [200]. This method is good for problems where (1) the task and the environment are extremely hard to model, (2) the state space is too large and (3) real-time computation is critical.

This classification may not be exhaustive. This book considers methods in Category 2 and Category 3 . The existing methods in designing the three components will be reviewed in the following sections.

\subsubsection{KNOWLEDGE}

As illustrated in Fig. 1.2a, knowledge may contain both raw data (e.g., measurementaction pairs $(\pi, u)$ ) and the system principles extracted from raw data (e.g., the cost $\mathcal{J}$ and the model $\mathcal{M})$. The former is also called model-free knowledge, while the latter is called model-based knowledge. For model-free knowledge, the measurement in the raw data may contain (1) system states and (2) the instant cost evaluation on current states and actions. In the following discussion, we call $(\pi, u)$ the state-actioncost pair, since it is a collection of system states, the corresponding desired actions demonstrated by human or calculated by a solver, and the instant cost evaluated on current states and actions. For model-based knowledge, the cost $\mathcal{J}$ provides incentives for the robot to finish the desired task efficiently as well as to interact safely with other agents in the system, while the model $\mathcal{M}$ should be designed in order to match the ground truth behavior of the environment. The cost $\mathcal{J}$ is a function that assigns values to all measurement-action pairs, while the instant cost in raw data is just a "point-wise" run-time measurement. 
State-action-cost pairs

"All models are wrong, but some are useful."

George E. P. Box

Because of the complexity of the real world, a manual-designed model is usually a subjective simplification of the actual system. On the other hand, raw data is an objective observation of the world, which usually contains more comprehensive information than a simplified extracted model. Recently, many data mining methods have been developed, trying to recover useful information from raw measurement data. Naturally, the first and critical step for data mining is to construct a database as comprehensive as possible to fully embed the system property.

In robotics, the state-action-cost pairs provide a straightforward encoding of cluttered data. These pairs describe the evolutional system dynamics, the desired policy principle and the hidden cost mechanism implicitly. The state variables are the smallest possible subset of system variables that can represent the entire system condition at any given time. The action is the control command that can be applied on the system to influence the evolution of the system. The instant cost is a quantitative description of the goodness or badness of the current state and action. For a rational agent, it is supposed to be an optimizer which tends to achieve the highest rewards or the lowest costs by taking appropriate actions.

These data, i.e., the state-action-cost pairs, can be acquired by human demonstration or by robot self-exploration. In the demonstration approach, a human operator demonstrates the desired action w.r.t. a specific state, and labels the current condition with a corresponding cost value. In the exploration approach, the robot autonomously explores the state space by following some heuristic rules. The former approach collects more biased samples which contain human intention of optimality. This sampling bias might concentrate the learning in a limited region and boost the learning convergence. The latter approach, on the other hand, involves more randomness and explores broader regions. It provides more implementation flexibility since no human demonstration is required.

\section{Cost}

Cost $\mathcal{J}$ is a function on the state and action of the robot, which encodes a form of internal incentives for the robot to finish certain tasks. A rational robot always seeks to minimize the cost. The cost can either be a static cost or a dynamic cost. Static cost is a function on current state and action, which can be measured as the instant cost in the raw data discussed earlier. Dynamic cost is a function on a trajectory, i.e., a sequence of future states and actions, which is a discounted sum of all static costs 
along the trajectory. ${ }^{8}$ Static cost includes runtime cost (e.g., the cost incurred before the completion of the task) and terminal cost (e.g., the cost related to the completion of the task). If the process never ends, there can be no terminal cost. For example, there is no terminal cost during lane following for automated vehicles. Dynamic cost can be summed either for a fixed time horizon (finite time or infinite time) or for a fixed task cycle. Fixed time horizon is adopted in linear quadratic regulator, preview control and receding horizon control. Fixed task cycle optimization is also called "minimum time problem" as the terminal time is treated as a decision variable. Fixed task cycle optimization is usually adopted in trajectory planning with fixed targets. The cost $\mathcal{J}$ will refer to the dynamic cost in the following discussion.

For model-based methods, the cost can either be designed or learned, with different levels of details. In Category 1 , the cost is explicitly specified by a designer to achieve certain design objectives. However, for complicated tasks, it is hard for a human designer to construct desired cost functions, while it is easier to let the robot learn from experience or learn from others. In Category 2, the cost is obtained from data, i.e., the state-action-cost pairs discussed above. In particular, model-based reinforcement learning allows the robots to learn static cost from past experience, which can then be combined to formulate dynamic cost. Inverse reinforcement learning allows the robot to infer the cost $\mathcal{J}$ from some observed policy $\hat{g}(\cdot \mid \mathcal{J}, \mathcal{M})$, e.g., from an expert. The design of $\mathcal{J}$ will be discussed in the following chapters w.r.t. specific applications.

\section{Model}

Since the robot model is usually known in advance, we focus on the models of other intelligent entities in the environment, especially humans. As pointed out in [43], the biggest challenge in human-robot collaboration comes from human factors. To make co-robots human-friendly, human behavior needs to be modeled, learned, and predicted [14, 32, 176, 230]. The following three kinds of models are frequently used to describe human behaviors.

1. Reactive model.

Under a reactive model, human dynamics are described using ordinary differential or difference equations. The inputs to the model are the identified features that affect human behaviors [191], while the output is the human motion.

2. Rational model.

Under a rational model, a human is treated as a cost minimizer [160, 217], whose cost function depends on both his or her behavior and the robot's behavior. The human will reason his or her best action given his or her belief in the robot's behavior.

\footnotetext{
${ }^{8}$ In the MDP context, the static cost is equivalent to the reward function and the dynamic cost is equivalent to the value function or the $Q$ function.
} 
3. Bayesian model.

Under a Bayesian model, a human is treated as a stochastic agent [215]. A Bayesian network [170, 179] is associated with his or her decisions, i.e., the human's motion follows a probabilistic distribution conditioned on the robot's behavior.

It is usually hard to obtain the cost function or probability distribution that precisely describes human behavior in sophisticated problems. Moreover, it is dangerous to assume that a human will always adapt his or her motion to a robot's motion, as it intrinsically implies that the robot can "control" the human [20]. Since no universal model exists that can precisely describe human behavior, learning of human behaviors is necessary.

\subsubsection{LOGIC}

As discussed in section 1.3.1 and illustrated in Fig. 1.3, there are two kinds of logic, i.e., model-based logic and model-free logic. The model-based logic assumes that the system follows some hidden deterministic rules. One deterministic rule can be that agents are rational. Under the rationality assumption, the control policy is designed to be an optimizer which explicitly calculates optimal actions for the robot to achieve optimal performance. The optimal performance is described by minimal cost in this book. The cost metric as a part of knowledge needs delicate design as discussed in section 1.3.2. In contrast, the model-free logic regards the system as a stochastic process, where the control policy is nothing but a regression function between inputs (states) and outputs (actions). This regression function can be approximated purely by data mining, or modeled partly by system analysis. This section discusses methods to design model-based logic. Learning of model-free logic will be discussed in section 1.3.4.

The fundamental problem of a model-based logic is to find the minimum in the cost $\mathcal{J}$, given the data $\pi$ and the model $\mathcal{M}$. In engineering applications, as the problems are complicated and in large scale, minimum seeking is non-trivial and various levels of approximations are needed. A model-based explicit policy is suitable for well-defined tasks and environments, however, hard to cover a variety of complicated scenarios that co-robots may face. This book focuses on model-based implicit policies which require online optimization. As we focus on physical movements in robot behaviors, the behavior design problem is essentially a motion planning problem. Depending on the look-ahead horizon of the dynamic cost, there are two kinds of motion planners, i.e., global planner (or long-term planner) and local planner (or short-term planner).

\section{Global planner}

A global planner evaluates the dynamic cost in a relatively long time horizon. The horizon may go to infinity. A comprehensive model of the environment is needed. 
The existing global motion planning methods fall into two categories: planning by construction and planning by modification. Planning by construction refers to methods that extend a trajectory by attaching new points to it until the target point is reached. Search-based methods such as A* or D* search [195] and sampling-based methods such as rapidly-exploring random tree $[105,108]$ are typical planning-byconstruction methods. Planning by modification refers to methods that perturb an existing trajectory so that the desired property is obtained. Optimization-based motion planning methods belong to this category. Gradient descent performs the perturbation or modification of the trajectory when solving the optimization $[90,189,56]$. Every type of methods has pros and cons. The trajectories planned by construction are more feasible than trajectories planned by modification. However, as the space is usually discretized during trajectory construction, the constructed trajectories are not as smooth as the trajectories planned by modification. This book focuses on optimization-based methods. If the environment is perfectly known without any uncertainty in advance, the global planner is capable of finding the global optimal trajectory. However, in practice, it is computationally expensive to build the model of the environment given the limited sensing ability and computational resource.

\section{Local planner}

A local planner tries to only plan a few steps ahead based on the limited knowledge of the environment obtained by current sensory data. Such planners require less computation power, and are good choices for robots with limited sensing abilities. An extreme case of a local planner is the reactive controller where the planning horizon reduces to one. There are usually closed-form solutions for reactive controllers. A closed-form solution of reactive control is a direct mapping from the sensory data to the control, which can greatly relieve the computation burden. ${ }^{9}$ Existing reactive methods include potential field method [95], barrier certificate [12], sliding mode method [74], and a family of biologically inspired methods [23]. However, as it only regulates the motion locally, a local planner is sensitive to local optima. It is possible for the robot to get stuck in some location and cannot reach the target. Thus, the global convergence to the target needs to be addressed when designing a local planner. In general, it is very hard to guarantee global convergence for local planners, as the system under consideration is nonlinear (as the robot motion is nonholonomic), time-varying (as the obstacles in the system are moving and deforming), and stochastic (as the information about the environment is limited).

To leverage the advantages of different planners, a global planner and a local planner can be combined in parallel, which will be discussed in Chapter 2.

\footnotetext{
${ }^{9} \mathrm{~A}$ reactive control policy is similar to an explicit policy in Fig. 1.3a in that they are both direct mapping from the sensory data to the control. However, only local information is considered in the reactive control policy, while global information is encoded in the explicit policy when it is computed in the design phase.
} 


\subsubsection{LEARNING PROCESS}

Learning process updates the knowledge or the logic according to the observed data. The ultimate goal of learning is to derive a better logic from the updates. As mentioned in section 1.3.1, there are model-based and model-free logics. In model-based approaches, learning process directly updates the knowledge, so that the logic can be updated based on physical principles and deductions [16]. In model-free approaches, the learning process directly updates the logic.

\section{Updating knowledge}

In a model-based approach, both the cost and the model can be updated. This book considers the learning on the model, which is a cognition skill. In particular, interpretation and prediction of human motions are considered. Learning on the model can be done both offline (in the training phase) and online (in the execution phase). Offline learning is usually used to classify human behaviors and to identify models that describe human behaviors as discussed in [19, 64, 178]. On the other hand, online learning is capable of adapting the offline learned models to humans' time-varying behaviors online as discussed in $[72,185]$. Methods to learn human model will be discussed in Chapter 5.

\section{Updating logic}

In model-based approaches, the logic is indirectly updated when the knowledge is updated. Such an approach is good for system transparency and interpretability, which is critical in many application domains, especially for industrial applications.

However, as robots are deployed to more sophisticated tasks, such as grasping dexterous objects and manipulating deformable objects, a pure model-based learning approach runs into its bottleneck. In these scenarios, either the system complexity goes beyond our capability to model, or the existing models cannot introduce satisfying results. On the other hand, along with the development of machine learning methods, especially the breakthrough of deep learning in recent years [110], people realize that: although human cognition process is complicated to model, it could be alternatively regressed by high dimensional parametric functions given a large amount of training data [157]. With this observation, many model-free learning approaches have been proposed recently, trying to teach robots new skills from data, instead of modeling those skills from principles. In the training stage, thousands or even millions of demonstration data is collected either by offline expert demonstration (imitation learning [24, 8], learning from demonstration [15, 17]), or by online trial and error (model-free reinforcement learning $[155,194])$. The collected samples are then utilized to fit an optimal control policy $g$ such that $u=g(\pi)$. These model-free learning approaches have universal structure and common training process, which can be quickly deployed to various scenarios. However, many of them 
are working in a black box manner. As a consequence, it can be hard to interpret the outcomes of model-free learning. The stability of the learning system is also difficult to evaluate. Therefore, model-free learning are usually deployed for high-level tasks (e.g., vision detection), while the low-level implementation (e.g., motor servoing) is still based on model-based learning approaches. This hierarchical structure achieves a balance between performance and stability of the overall system. It is notable that for non-safety-critical scenarios, end-to-end learning [113, 31] is favored, where the hierarchical structures of systems are embedded in a single framework of deep neural networks, and thus the maximum potential of neural networks is exploited.

The model-free learning approaches, together with the model-based learning approaches which need online system identification, are called data-driven approaches in general. The learning methods will be discussed in Chapters 5 and 6.

\subsection{DESIGN OBJECTIVES}

Intelligent robots are multi-purposed complex entities. Their behavior systems (introduced earlier in section 1.3) need to be designed such that the robots are safe during human-robot interactions, efficient in task performance, able to imitate behaviors of other intelligent entities, dexterous to adapt to different environments and tasks, and cooperative with other intelligent entities. This book studies methodologies to achieve these five design objectives in Part II. The challenges and existing solutions to address these design objectives are discussed below.

\subsubsection{SAFETY}

Human safety is one of the biggest concerns in HRI [204]. Robots should be both intrinsically safe, e.g., minimizing impact if collision happens [79], and interactively safe, e.g., avoiding collision at the first place [216]. Interactive safety needs to be addressed in the context of decision making, motion planning and control of the robot during HRI.

The difficulty of designing safe robot behavior comes from (1) sophistication of human motion and (2) real-time implementation of the algorithm. Control theory offers plenty of methods to deal with disturbances. However, when humans are in the loop, the disturbances introduced by human motions are much more complicated. They are time varying with unknown models. Moreover, it seems counterproductive to view human motions as disturbances in the robot control, as the system should be human-oriented. Learning of human behaviors is needed to make robots compliant. Even with robots learning human behaviors, another concern is that a complex control algorithm may not be executed fast enough to ensure quick responses of robots in emergencies.

Conventional approach to address the interactive safety is conservative, which slows down the robot when humans are nearby, hence sacrifices productivity for the sake of safety. Less conservative methods in the context of obstacle avoidance have 
also been used to address safety in HRI, e.g., potential field methods [168, 95], sliding mode methods [74]. These two methods result in closed-form analytical control laws, but are not optimal. Some authors formulate the problem as an optimization or optimal control problem with hard constraints to represent the safety requirements [56]. Unfortunately, these non-convex optimization problems are generally hard to solve analytically [86]. Different approximations and numerical methods are used to obtain solutions $[189,195]$. However, these numerical methods may not be executed fast enough for timely responses in emergency situations.

Chapter 3 discusses the approaches that we developed for safe human-robot interaction. The problem is formulated as an optimization with hard safety constraints, which is non-convex. We then transform the non-convex state space constraint into a convex control space constraint using the idea of invariant set. In this way, the problem is transformed to a convex problem which can be solved efficiently online. This method can also take the uncertainty in the human behavior into account. It has been shown that the safety constraint will never be violated using the safe control method if the human behavior satisfies certain assumptions.

\subsubsection{EFFICIENCY}

Robot efficiency is essential to maximize task performance. Robots should understand the current situation, then plan and execute the proper actions to complete the task in minimum time. In human-involved dynamic environments, robot motion needs frequent replanning to cope with time-varying tasks. Hence, computation efficiency of the motion planning algorithms is critical to ensure real-time response of the robot.

The challenge of efficient motion planning results from the complexity of the planning problem, which may be highly nonlinear due to the dynamic constraints, and highly non-convex due to the constraints for obstacle avoidance. It is hard to solve the planning problem in real time.

This book focuses on optimization-based motion planning. There are various methods developed to deal with the non-linearity and non-convexity [163, 199] in optimization-based motion planning. One popular way is through convexification [210], e.g., transforming the non-convex problem into a convex one. Some authors tried to transform the non-convex problem to semidefinite programming [58]. Some authors proposed to introduce lossless convexification by augmenting the space $[9,83]$, and some authors proposed successive linear approximation to remove non-convex constraints $[141,142]$. However, the first method requires the cost function to be quadratic. The second approach depends highly on the linearity of the system and may not be able to handle various obstacles. Finally, the third approach may not generalize to non-differentiable problems. One of the most popular convexification methods is the sequential quadratic programming (SQP) [198, 213], which approximates the non-convex problem as a sequence of quadratic programming problems and solves them iteratively. The method has been successfully applied to offline robot motion planning [91, 189]. However, as SQP is a generic algorithm, the unique 
structure of the motion planning problems is neglected, which usually results in failure to meet the real-time requirement in engineering applications.

Chapter 4 discusses the approaches that we developed to ensure efficient robot motion planning during human-robot interactions. In practice, the cost function for motion planning is designed to be convex [221, 177], while the non-convexity mainly comes from the physical constraints, e.g., robot dynamics and collision avoidance. By exploiting the geometric structure of the problems, we propose the convex feasible set algorithm [127] and the slack convex feasible set algorithm [136] to handle motion planning problems with convex objective functions and non-convex constraints. It will be shown that these methods perform better than generic methods in robot motion planning in terms of efficiency.

\subsubsection{IMITATION}

Imitation is the ability of robots to reproduce human-like movements. On the one hand, by learning to mimic human behavior, robots can better predict human's future motion through mental simulation. Better prediction of human motion can lead to smoother human-robot interaction. On the other hand, robots can physically behave more like humans, so as to accomplish dexterous tasks which usually require human skills.

Take robotic precision assembly as an example. Tuning an appropriate force controller for a robot is usually a non-trivial task. In contrast, humans can accomplish assembly tasks compliantly with much less time and fewer trials. Human's inherent assembly skill is achieved by a form of internal control, which has been well tuned through our daily operation and experience. It is more intuitive and efficient to teach robots insertion skill by mimicking human's compliant motions, compared to designing a new controller from scratch [206, 114, 44, 101].

In general, the challenges for robots to mimic human behavior come from two aspects. First, it is difficult to select the proper variables to sufficiently and efficiently depict human behavior, while those variables should be observable and measurable by available sensors. Second, it is difficult to properly encode the measured human behaviors, and to reproduce human-like motions in novel scenarios. There are specific challenges associated with imitation for prediction [201, 224] and imitation for action [39, 40, 187]. Regarding imitation for prediction, the major problem is homogeneity of the learned models, which ignores individual differences. Hence, to make accurate "personalized" predictions, online adaptation of those models are necessary. Regarding imitation for action (or skill learning), few of those existing methods are implementable on industrial robots. Unlike collaborative robots, industrial robots are highly rigid because of large gear reduction ratio on the drive train. For assembly tasks, it implies that there is no compliance on the industrial robot's mechanism to assist assembly. Besides, the clearance requirement for industrial assembly is much more strict. Therefore, new schemes which are implementable on industrial robots need to be developed to support imitation learning. 
Chapter 5 discusses methods to (1) learn individualized human behavior models so as to make better predictions; and (2) teach industrial robots assembly skills from human demonstration with varying admittance. For prediction, we introduce online learning. For skill learning, we introduce a model-free framework with guaranteed stability from Lyapunov analysis.

\subsubsection{DEXTERITY}

Dexterity is the ability of robots to change its nominal motion patterns to adapt to the change of its surrounding environment or task requirements.

Although there are numerous approaches for robot learning, most of them follow a similar pipeline: learning the logic in the training stage, and then applying the learned logic in new scenarios. It is implicitly assumed that the learned logic is universally applicable. However, it remains challenging to verify that the learned logic is truly universal and dexterous. To make robots dexterous, it will be beneficial to first understand the dexterity of human and then equip robots with the same ability.

Many studies in cognitive science shows that humans can make dexterous decisions w.r.t. various scenarios through analogical reasoning [42, 164, 116]. Given a new scenario, humans will try to find its correlation with past scenarios, and transfer previous problem-solving actions to the new situation. With this analogy approach, the problem can be solved without understanding the universal policy or causality behind the system. This analogy approach also enables humans to learn efficiently from small data samples.

Chapter 6 discusses an analogy-based learning approach to make robots dexterous. Unlike traditional model-based or model-free approaches, which try to formulate control policies, this new approach focuses on finding correlations among scenarios. This approach has two stages: the reminding stage and the transferring stage. During the reminding stage, the similarity between the past scenarios and the current scenario will be calculated. The most similar pair will be selected and a mapping function between the two scenarios will be constructed by non-rigid registration. During the transferring stage, the past actions will be transformed by the mapping function to generate new actions in the current situation. This approach is plausible for applications which are hard to model or to collect data from. More properties and advantages of analogy-based learning will be introduced in details in Chapter 6.

\subsubsection{COOPERATION}

Cooperation is the ability of robots to achieve a mutual goal together with other intelligent entities (e.g., humans or other robots) without centralized coordinations.

Cooperation is hard to achieve mainly due to information asymmetry [175], i.e., the parameter in the behavior system of one agent is unknown to another agent. Information asymmetry is common in HRI. From the agent perspective, most learning and control strategies follow a centralized design philosophy, which adjusts the 
agent's estimation if its observation deviates from its prediction. This strategy is suitable in the single agent case, since the deviation can only be caused by the agent's wrong estimation. However, in a multi-agent system, where each agent acts on its estimation of the unknowns, the gap between an agent's observation and prediction is not only caused by the agent's wrong estimation (wrong prediction), but also other agents' wrong estimations and subsequent improper actions (deviated observations). Interactions and other agents' sub-optimality need to be considered explicitly in agent behavior design.

Conventionally, to compensate information asymmetry during online execution, distributed control laws are designed for each robot agent [180]. Following the control laws specified by the intelligent designer, who has an omniscient perspective, the agents do not need much intelligence. Although it is not explicitly stated that the control laws are globally known, the effectiveness of the controllers is based on the confidence that the designed control laws are perfectly followed by all agents. In this case, an agent just needs to follow its own control law, while other agents will take care of everything else by following their designed control laws. This strategy usually works well on problems with predefined environment and interaction patterns, but has limited extendability, as it is hard to design a universal control law that leads to desired results in a wide variety of situations, especially when the system topology is time-varying (interactions are happening among different agents at different times).

Chapter 7 discusses two ways to encourage cooperation. The first method introduces compensations of other agents' wrong estimations in the ego agent's learning strategy. In this way, even without explicit communication, the multi-agent system can still achieve the mutual goal. The second method allows agents to communicate with each other to resolve the conflicts. A conflict resolution mechanism is developed to form a consensus.

\subsection{SYSTEM EVALUATION}

The designed robot behaviors need to be evaluated in human-robot systems. The evaluation can be performed theoretically as well as experimentally.

\subsubsection{THEORETICAL EVALUATION}

During theoretical analysis, the questions to answer are:

1. Is the logic optimal and safe?

2. Will the learning process generate converging sequences to the ground truth?

3. Will the designed behaviors lead to desired outcomes of the multi-agent system? 
The first two questions are modular-wise. The third question is system-wise which concerns with the stability, robustness stability and optimality of the closed loop system, e.g., whether the closed loop multi-agent system is self-organized [140]. System level analysis is challenging due to (1) the complication of interactions among different agents, (2) the difficulty in justifying the assumptions on human behavior; (3) the insufficiency of existing tools in game theory to analyze suboptimal agents. ${ }^{10}$

\subsubsection{EXPERIMENTAL EVALUATION}

In addition to theoretical analysis, experiments should be conducted to evaluate the performance of the designed robot behaviors. However, to protect human subjects, it is desirable if we can separate human subjects and the robot physically during the early phase of deployment. Figure 1.4 shows five different platforms that have been developed for experimental evaluations.

The first platform is the virtual reality-based human-in-the-loop platform. The robot's motion is simulated in a robot simulator. A human subject observes the robot movement through the virtual reality display (e.g., virtual reality glasses, augmented reality glasses or monitors). The reaction of the human subject is captured by sensors (e.g., Kinect or touchpad). The sensor data is then sent to the robot behavior system to compute the desired control input. The advantage of this platform is that it is safe to human subjects and convenient for idea testing. The disadvantage is that the robot simulator may neglect dynamic details of the physical robot, hence reducing the reliability of the results.

The second platform is the virtual reality-based hardware-in-the-loop platform. Its difference from the first platform is that the robot motion is no longer simulated, but directly measured from the robot hardware. This solves the problem of dynamic mis-match that the robot simulator may have. However, as the interaction happens virtually, the human subjects may not react in the same way that they do with real robots.

The third platform is the dummy-robot interaction platform. The interaction happens physically between the dummy and the robot. The human directly observes the robot motion and controls the dummy to interact with the robot. As there are physical interactions, an emergency check module should be added to ensure safety. The advantage of this platform is that it is safe to human subjects, while it is able to test interactions physically. However, the disadvantage is that the dummy usually has fewer degrees of freedom than a human subject.

The fourth platform is the robot-robot interaction platform. Each robot regards the other as a "human". In general, such a platform allows us to inquire whether the designed behavior system can cope with intelligent entities other than humans. Moreover, it can examine whether the multi-agent system consisting of those intelligent robots are stable or not.

\footnotetext{
${ }^{10}$ The basic assumption in game theory is that all agents are rational, e.g., always behaving optimally. However, the logic that we designed for the robot may not be optimal.
} 


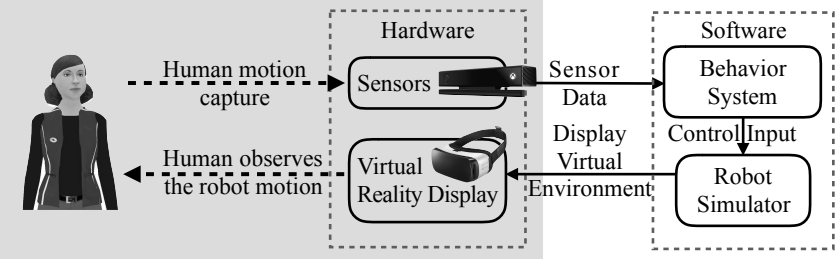

(a) Virtual reality-based human-in-the-loop platform.

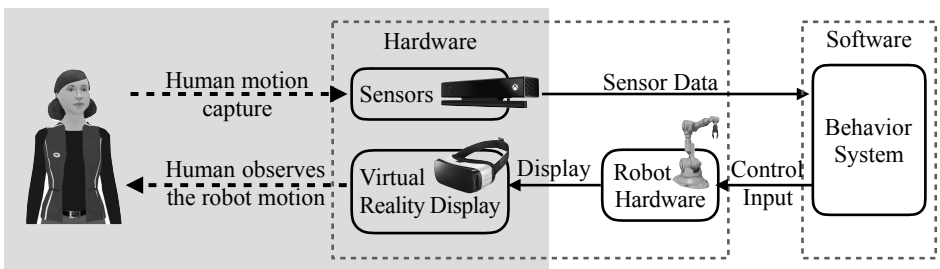

(b) Virtual reality-based hardware-in-the-loop platform.

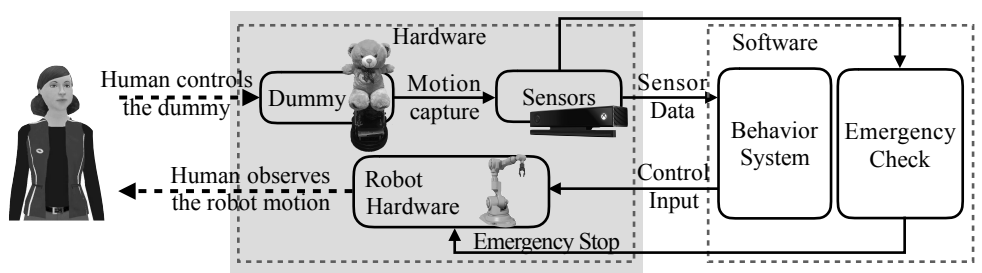

(c) Dummy-robot interaction platform.

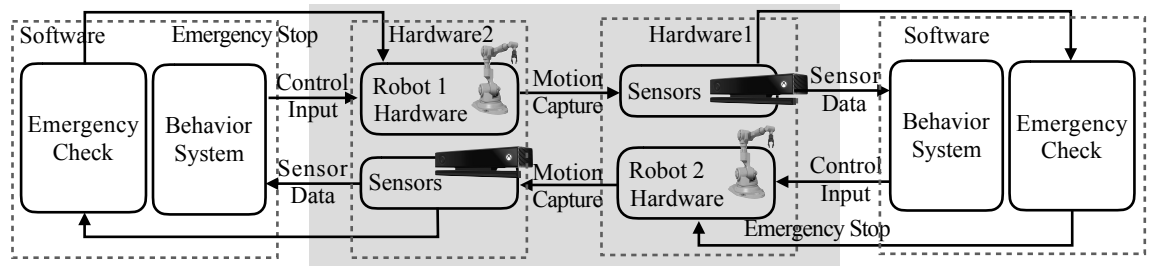

(d) Robot-robot interaction platform.

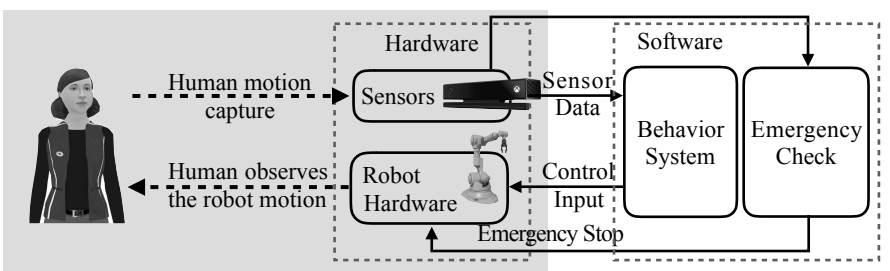

(e) Human-robot interaction platform.

Figure 1.4: The evaluation platforms. 
The fifth platform is the human-robot interaction platform where human subjects directly interact with robots. Such platform should be designed to illustrate the performance of the robot in real tasks.

In Part III of the book, evaluation results on those platforms will be discussed.

\subsection{OUTLINE OF THE BOOK}

This book aims to establish a set of methodologies for robot behavior design in order to maximize their performance in human-involved dynamic uncertain environments. The emphases of design objectives are safety, efficiency, imitation, dexterity, and cooperation.

As illustrated in Fig. 1.5, the remainder of the book is organized as follows.

Part I. Introduction

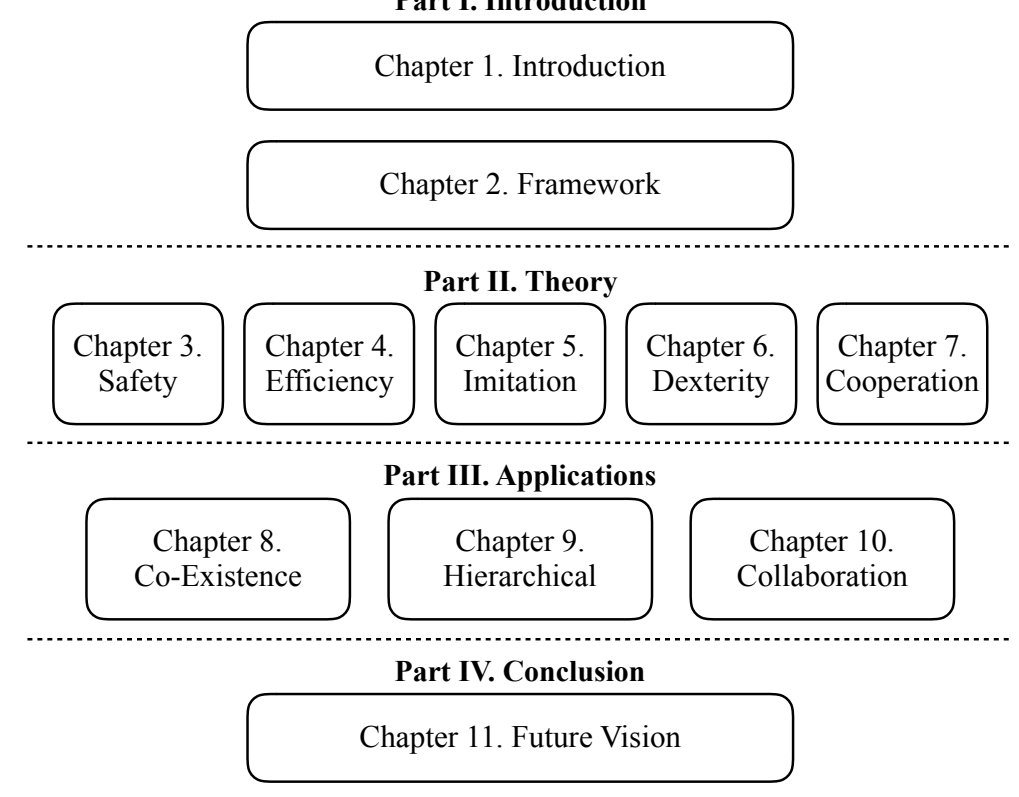

Figure 1.5: Overview of the book content.

Part I is for introduction. Chapter 2 overviews the mathematical methods for robot behavior design in a multi-agent framework.

Part II discusses theory. Chapter 3 is for safety. Chapter 4 is for efficiency. Chapter 5 is for imitation. Chapter 6 is for dexterity. Chapter 7 is for cooperation.

Part III discusses applications. Chapter 8 discusses space-sharing human-robot interactions, such as the interaction between industrial co-robots and human workers, 
and the interaction between autonomous vehicles and other road participants. Chapter 9 discusses hierarchical human-robot interactions where a robot learns skills from human teachers. Chapter 10 discusses time-sharing human-robot interactions where a robot and a human collaborate to finish a joint task.

Part IV discusses future vision and concludes the book.

Each chapter is intended to be self-contained. Some of the work has been published in $[117,118,119,120,124,127,128,130,131,132,133,134,135,136,137$, $138,139,206,207,208,209]$. 


\section{References}

1. Baxter from Rethink Robotics.

2. UR5 from Universal Robotics.

3. Kawada's NextAge robot, 2011.

4. Volvo collision avoidance features: Initial results. Technical Report 5, Highway Loss Data Institute, 2012.

5. Working with robots: Our friends electric. The Economist, Sep. 5, 2013. https:// www.economist.com/technology-quarterly/2013/09/05/our-friends-electric, 2013.

6. Taxonomy and definitions for terms related to on-road motor vehicle automated driving systems, 2014.

7. Driverless car market watch, 2015.

8. Pieter Abbeel and Andrew Y Ng. Apprenticeship learning via inverse reinforcement learning. In Proceedings of the International Conference on Machine learning (ICML), page 1, 2004.

9. Behçet Açımeşe, John M Carson, and Lars Blackmore. Lossless convexification of nonconvex control bound and pointing constraints of the soft landing optimal control problem. IEEE Transactions on Control Systems Technology, 21(6):2104-2113, 2013.

10. Nadav Aharony, Wei Pan, Cory Ip, Inas Khayal, and Alex Pentland. Social fmri: Investigating and shaping social mechanisms in the real world. Pervasive and Mobile Computing, 7(6):643-659, 2011.

11. Baris Akgun, Maya Cakmak, Jae Wook Yoo, and Andrea Lockerd Thomaz. Trajectories and keyframes for kinesthetic teaching: A human-robot interaction perspective. In Proceedings of the ACM/IEEE International Conference on Human-Robot Interaction, pages 391-398, 2012.

12. Aaron D Ames, Xiangru Xu, Jessy W Grizzle, and Paulo Tabuada. Control barrier function based quadratic programs for safety critical systems. IEEE Transactions on Automatic Control, 62(8):3861-3876, 2017.

13. Tanya M Anandan. Major robot OEMs fast-tracking cobots, 2014.

14. T Arai, R Kato, and M Fujita. Assessment of operator stress induced by robot collaboration in assembly. CIRP Annals-Manufacturing Technology, 59(1):5-8, 2010.

15. Brenna D Argall, Sonia Chernova, Manuela Veloso, and Brett Browning. A survey of robot learning from demonstration. Robotics and Autonomous Systems, 57(5):469483, 2009.

16. Christopher G Atkeson, Eric W Aboaf, Joseph McIntyre, and David J Reinkensmeyer. Model-based robot learning. 1988.

17. Christopher G Atkeson and Stefan Schaal. Robot learning from demonstration. In ICML, volume 97, pages 12-20, 1997.

18. Seyed Azimi, Gaurav Bhatia, Ragunathan Rajkumar, and Priyantha Mudalige. Reliable intersection protocols using vehicular networks. In 2013 ACM/IEEE International Conference on Cyber-Physical Systems, pages 1-10.

19. Joonbum Bae and Masayoshi Tomizuka. Gait phase analysis based on a hidden Markov model. IEEE/ASME Transactions on Mechatronics, 21(6):961-970, 2011.

20. Lisanne Bainbridge. Ironies of automation. Automatica, 19(6):775-779, 1983.

21. Albert Bandura. Social cognitive theory: An agentic perspective. Annual Review of Psychology, 52(1):1-26, 2001. 
22. Tamer Basar and Geert Jan Olsder. Dynamic Noncooperative Game Theory, volume 200. London: Academic Press, 1995.

23. George A Bekey. Autonomous Robots: From Biological Inspiration to Implementation and Control. MIT Press, 2005.

24. Darrin C Bentivegna, Christopher G Atkeson, and Gordon Cheng. Learning tasks from observation and practice. Robotics and Autonomous Systems, 47(2-3):163-169, 2004.

25. Charles R Berger and Richard J Calabrese. Some explorations in initial interaction and beyond: Toward a developmental theory of interpersonal communication. Human Communication Research, 1(2):99-112, 1975.

26. Dimitris Bertsimas, Vishal Gupta, and Ioannis Ch Paschalidis. Data-driven estimation in equilibrium using inverse optimization. Mathematical Programming, pages 1-39, 2014.

27. Jeff A Bilmes et al. A gentle tutorial of the em algorithm and its application to parameter estimation for gaussian mixture and hidden markov models. International Computer Science Institute, 4(510):126, 1998.

28. Christopher M Bishop. Pattern recognition and machine learning, volume 1. Springer: New York, 2006.

29. Paul T Boggs and Jon W Tolle. Sequential quadratic programming. Acta Numerica, 4:1-51, 1995.

30. Jeannette Bohg, Antonio Morales, Tamim Asfour, and Danica Kragic. Data-driven grasp synthesis-a survey. IEEE Transactions on Robotics, 30(2):289-309, 2014.

31. Mariusz Bojarski, Davide Del Testa, Daniel Dworakowski, Bernhard Firner, Beat Flepp, Prasoon Goyal, Lawrence D Jackel, Mathew Monfort, Urs Muller, Jiakai Zhang, et al. End to end learning for self-driving cars. arXiv preprint arXiv:1604.07316, 2016.

32. Paulo Vinicius Koerich Borges, Nicola Conci, and Andrea Cavallaro. Video-based human behavior understanding: a survey. IEEE Transactions on Circuits and Systems for Video Technology, 23(11):1993-2008, 2013.

33. S Bouchard. With two arms and a smile, Pi4 Workerbot is one happy factory bot. IEEE Spectrum, 2011.

34. Cynthia Breazeal. Social interactions in hri: the robot view. IEEE Transactions on Systems, Man, and Cybernetics-Part C: Applications and Reviews, 34(2):181-186, 2004.

35. Douglas A Bristow, Marina Tharayil, and Andrew G Alleyne. A survey of iterative learning control. IEEE Control Systems, 26(3):96-114, 2006.

36. Peter Brook, Matei Ciocarlie, and Kaijen Hsiao. Collaborative grasp planning with multiple object representations. In Robotics and Automation (ICRA), 2011 IEEE International Conference on, pages 2851-2858. IEEE, 2011.

37. Lawrence D Burns. Sustainable mobility: a vision of our transport future. Nature, 497(7448):181-182, 2013.

38. Richard H Byrd, Jorge Nocedal, and Richard A. Waltz. pages 35-59. Springer US, Boston, MA.

39. S Calinon and A Billard. Learning of Gestures by Imitation in a Humanoid Robot. In Imitation and Social Learning in Robots, Humans and Animals: Behavioural, Social and Communicative Dimensions, pages 153-177. Cambridge University Press, 2007. K Dautenhahn and CL Nehaniv (Eds).

40. Sylvain Calinon, Florent D'halluin, Eric L Sauser, Darwin G Caldwell, and Aude G Billard. Learning and reproduction of gestures by imitation. IEEE Robotics and Automation Magazine, 17(2):44-54, 2010. 
41. Olivier Cappé, Eric Moulines, and Tobias Rydén. Inference in Hidden Markov Models. Springer Science \& Business Media, New York, 2006.

42. Jaime $\mathrm{G}$ Carbonell. Learning by analogy: Formulating and generalizing plans from past experience. In Machine Learning, Volume I, pages 137-161. Springer, Berlin, Heidelberg, 1983.

43. George Charalambous, Sarah Fletcher, and Philip Webb. Human-automation collaboration in manufacturing. In Contemporary Ergonomics and Human Factors 2013, volume 59, pages 59-66. ROUTLEDGE in association with GSE Research, 2013.

44. Yutuo Chen, Xuli Han, Minoru Okada, Yu Chen, and Fazel Naghdy. Intelligent robotic peg-in-hole insertion learning based on haptic virtual environment. In Computer-Aided Design and Computer Graphics, 2007 10th IEEE International Conference on, pages 355-360. IEEE, 2007.

45. Yujiao Cheng, Weiye Zhao, Changliu Liu, and Masayoshi Tomizuka. Human motion prediction using adaptable neural networks. arXiv:1810.00781, 2018.

46. Rahul Chipalkatty, Hannes Daepp, Magnus Egerstedt, and Wayne Book. Human-inthe-loop: Mpc for shared control of a quadruped rescue robot. In Proceedings of the IEEE/RSJ International Conference on Intelligent Robots and Systems (IROS), pages 4556-4561, 2011.

47. Haili Chui and Anand Rangarajan. A new point matching algorithm for non-rigid registration. Computer Vision and Image Understanding, 89(2):114-141, 2003.

48. ChuiDataset. Data set for testing non-rigid registration. http://legacydirs . umiacs . umd . edu/ \%7Ezhengyf/PointMatching, 2000.

49. Hao Dang and Peter K Allen. Semantic grasping: Planning robotic grasps functionally suitable for an object manipulation task. In Intelligent Robots and Systems (IROS), 2012 IEEE/RSJ International Conference on, pages 1311-1317. IEEE, 2012.

50. Kenny Daniel, Alex Nash, Sven Koenig, and Ariel Felner. Theta*: Any-angle path planning on grids. Journal of Artificial Intelligence Research, 39:533-579, 2010.

51. Kerstin Dautenhahn. Robots we like to live with?!- a developmental perspective on a personalized, life-long robot companion. In Proceedings of the IEEE International Workshop on Robot and Human Interactive Communication, pages 17-22, 2004.

52. Kerstin Dautenhahn. Socially intelligent robots: Dimensions of human-robot interaction. Philosophical Transactions of the Royal Society B: Biological Sciences, 362(1480):679-704, 2007.

53. Arthur P Dempster, Nan M Laird, and Donald B Rubin. Maximum likelihood from incomplete data via the em algorithm. Journal of the Royal Statistical Society. Series B (Methodological), pages 1-38, 1977.

54. Renaud Detry, Carl Henrik Ek, Marianna Madry, Justus Piater, and Danica Kragic. Generalizing grasps across partly similar objects. In Robotics and Automation (ICRA), 2012 IEEE International Conference on, pages 3791-3797. IEEE, 2012.

55. M Bernardine Dias, Balajee Kannan, Brett Browning, E Gil Jones, Brenna Argall, M Freddie Dias, Marc Zinck, Manuela M Veloso, and Anthony J Stentz. Sliding autonomy for peer-to-peer human-robot teams. In Proceedings of the Intelligent Conference on Intelligent Autonomous Systems (IAS), pages 332-341, 2008.

56. Noel E Du Toit and Joel W Burdick. Robot motion planning in dynamic, uncertain environments. IEEE Transactions on Robotics, 28(1):101-115, 2012.

57. David Eberly. Robust Computation of Distance Between Line Segments, 2015.

58. Gabriele Eichfelder and Janez Povh. On the set-semidefinite representation of nonconvex quadratic programs over arbitrary feasible sets. Optimization Letters, 7(6):13731386, 2013. 
59. M Elhenawy, AA Elbery, AA Hassan, and HA Rakha. An intersection game-theorybased traffic control algorithm in a connected vehicle environment. In 2015 IEEE International Conference on Intelligent Transportation Systems, pages 343-347.

60. Martin Ester, Hans-Peter Kriegel, Jörg Sander, Xiaowei Xu, et al. A density-based algorithm for discovering clusters in large spatial databases with noise. In $K d d$, volume 96, pages 226-231, 1996.

61. Dave Ferguson and Anthony Stentz. Field d*: An interpolation-based path planner and replanner. In Robotics Research, pages 239-253. Springer, 2007.

62. Carlo Ferrari and John Canny. Planning optimal grasps. In Robotics and Automation, 1992. Proceedings, 1992 IEEE International Conference on, pages 2290-2295. IEEE, 1992.

63. Terrence Fong, Illah Nourbakhsh, and Kerstin Dautenhahn. A survey of socially interactive robots. Robotics and Autonomous Systems, 42(3):143-166, 2003.

64. Katerina Fragkiadaki, Sergey Levine, Panna Felsen, and Jitendra Malik. Recurrent network models for human dynamics. In Proceedings of the IEEE International Conference on Computer Vision (ICCV), pages 4346-4354, 2015.

65. Antonio Franchi, Cristian Secchi, Hyoung Il Son, Heinrich H Bülthoff, and Paolo Robuffo Giordano. Bilateral teleoperation of groups of mobile robots with timevarying topology. IEEE Transactions on Robotics, 28(5):1019-1033, 2012.

66. Jonathan D Gammell, Siddhartha S Srinivasa, and Timothy D Barfoot. Informed rrt*: Optimal sampling-based path planning focused via direct sampling of an admissible ellipsoidal heuristic. In Intelligent Robots and Systems (IROS 2014), 2014 IEEE/RSJ International Conference on, pages 2997-3004. IEEE, 2014.

67. Song Ge, Guoliang Fan, and Meng Ding. Non-rigid point set registration with globallocal topology preservation. In Computer Vision and Pattern Recognition Workshops (CVPRW), 2014 IEEE Conference on, pages 245-251. IEEE, 2014.

68. Jeremy H Gillula, Haomiao Huang, Michael P Vitus, and Claire J Tomlin. Design of guaranteed safe maneuvers using reachable sets: Autonomous quadrotor aerobatics in theory and practice. In 2010 IEEE International Conference on Robotics and Automation, pages 1649-1654. IEEE, 2010.

69. Federico Girosi, Michael Jones, and Tomaso Poggio. Regularization theory and neural networks architectures. Neural Computation, 7(2):219-269, 1995.

70. Matthew Golub, Steven Chase, and M Yu Byron. Learning an internal dynamics model from control demonstration. In Proceedings of the International Conference on Machine Learning (ICML), pages 606-614, 2013.

71. D González, J Pérez, V Milanés, and F Nashashibi. A review of motion planning techniques for automated vehicles. IEEE Transactions on Intelligent Transportation Systems, 17(4):1135-1145, 2016.

72. Michael A Goodrich and Alan C Schultz. Human-robot interaction: A survey. Foundations and Trends in Human-Computer Interaction, 1(3):203-275, 2007.

73. Graham C Goodwin and Kwai Sang Sin. Adaptive Filtering Prediction and Control. Courier Dover Publications, 2013.

74. Luis Gracia, Fabricio Garelli, and Antonio Sala. Reactive siding-mode algorithm for collision avoidance in robotic systems. IEEE Transactions on Control Systems Technology, 21(6):2391-2399, 2013.

75. Leslie Greengard and John Strain. The fast gauss transform. SIAM Journal on Scientific and Statistical Computing, 12(1):79-94, 1991. 
76. Elena Gribovskaya, Abderrahmane Kheddar, and Aude Billard. Motion learning and adaptive impedance for robot control during physical interaction with humans. In Proceedings of the IEEE International Conference on Robotics and Automation (ICRA), pages 4326-4332, 2011.

77. Tianyu Gu, J Atwood, Chiyu Dong, JM Dolan, and Jin-Woo Lee. Tunable and stable real-time trajectory planning for urban autonomous driving. In Proceedings of the IEEE/RSJ International Conference on Intelligent Robots and Systems (IROS), pages 250-256, 2015.

78. Tianyu Gu, John M Dolan, and Jin-Woo Lee. Runtime-bounded tunable motion planning for autonomous driving. In Proceedings of the IEEE Intelligent Vehicles Symposium (IV), pages 1301-1306, 2016.

79. Sami Haddadin, A Albu-Schaffer, Oliver Eiberger, and Gerd Hirzinger. New insights concerning intrinsic joint elasticity for safety. In Proceedings of the IEEE/RSJ International Conference on Intelligent Robots and Systems (IROS), pages 2181-2187, 2010.

80. Sami Haddadin, Alin Albu-Schaffer, Alessandro De Luca, and Gerd Hirzinger. Collision detection and reaction: A contribution to safe physical human-robot interaction. In Proceedings of the IEEE/RSJ International Conference on Intelligent Robots and Systems (IROS), pages 3356-3363, 2008.

81. Sami Haddadin, Michael Suppa, Stefan Fuchs, Tim Bodenmüller, Alin Albu-Schäffer, and Gerd Hirzinger. Towards the robotic co-worker. In Robotics Research, volume 70, pages 261-282. Springer Berlin Heidelberg, 2011.

82. Christopher Harper and Gurvinder Virk. Towards the development of international safety standards for human robot interaction. International Journal of Social Robotics, 2(3):229-234, 2010.

83. Matthew W Harris and Behçet Açıkmeşe. Lossless convexification of non-convex optimal control problems for state constrained linear systems. Automatica, 50(9):23042311, 2014.

84. Peter E Hart, Nils J Nilsson, and Bertram Raphael. A formal basis for the heuristic determination of minimum cost paths. IEEE Transactions on Systems Science and Cybernetics, 4(2):100-107, 1968.

85. John A Hartigan and Manchek A Wong. Algorithm as 136: A k-means clustering algorithm. Applied Statistics, pages 100-108, 1979.

86. Richard F Hartl, Suresh P Sethi, and Raymond G Vickson. A survey of the maximum principles for optimal control problems with state constraints. SIAM Review, 37(2):181-218, 1995.

87. Nicholas J Higham. Analysis of the Cholesky decomposition of a semi-definite matrix. Oxford University Press, 1990.

88. Gerd Hirzinger, A Albu-Schaffer, M Hahnle, Ingo Schaefer, and Norbert Sporer. On a new generation of torque controlled light-weight robots. In Proceedings of the IEEE International Conference on Robotics and Automation (ICRA), volume 4, pages 33563363, 2001.

89. Robert Hof. Toyota: 'guardian angel' cars will beat self-driving cars. Forbes, 2016.

90. Thomas M Howard, Colin J Green, and Alonzo Kelly. Receding horizon modelpredictive control for mobile robot navigation of intricate paths. In Field and Service Robotics, pages 69-78, 2010.

91. Tor Arne Johansen, Thor I Fossen, and Stig P Berge. Constrained nonlinear control allocation with singularity avoidance using sequential quadratic programming. IEEE Transactions on Control Systems Technology, 12(1):211-216, 2004.

92. Sertac Karaman and Emilio Frazzoli. Incremental sampling-based algorithms for optimal motion planning. Robotics Science and Systems VI, 104:2, 2010. 
93. Lydia E Kavraki, Petr Svestka, J-C Latombe, and Mark H Overmars. Probabilistic roadmaps for path planning in high-dimensional configuration spaces. IEEE Transactions on Robotics and Automation, 12(4):566-580, 1996.

94. S Mohammad Khansari-Zadeh, Klas Kronander, and Aude Billard. Modeling robot discrete movements with state-varying stiffness and damping: A framework for integrated motion generation and impedance control. Proceedings of Robotics: Science and Systems X (RSS 2014), 2014.

95. Oussama Khatib. Real-time obstacle avoidance for manipulators and mobile robots. The International Journal of Robotics Research, 5(1):90-98, 1986.

96. Byoung-Ho Kim, Sang-Rok Oh, Byung-Ju Yi, and Il Hong Suh. Optimal grasping based on non-dimensionalized performance indices. In Intelligent Robots and Systems, 2001. Proceedings. 2001 IEEE/RSJ International Conference on, volume 2, pages 949 956. IEEE, 2001.

97. Ulrich Klank, Dejan Pangercic, Radu Bogdan Rusu, and Michael Beetz. Real-time cad model matching for mobile manipulation and grasping. In Humanoid Robots, 2009. Humanoids 2009. 9th IEEE-RAS International Conference on, pages 290-296. IEEE, 2009.

98. Jens Kober and Jan Peters. Reinforcement learning in robotics: A survey. In Reinforcement Learning, pages 579-610. Springer, 2012.

99. Thomas Kollar and Nicholas Roy. Trajectory optimization using reinforcement learning for map exploration. The International Journal of Robotics Research, 27(2):175196, 2008.

100. Kyoungchul Kong, Joonbum Bae, and Masayoshi Tomizuka. Control of rotary series elastic actuator for ideal force-mode actuation in human-robot interaction applications. IEEE/ASME Transactions on Mechatronics, 14(1):105-118, 2009.

101. Klas Kronander, Etienne Burdet, and Aude Billard. Task transfer via collaborative manipulation for insertion assembly. In Workshop on Human-Robot Interaction for Industrial Manufacturing, Robotics, Science and Systems. Citeseer, 2014.

102. Jörg Krüger, Terje K Lien, and Alexander Verl. Cooperation of human and machines in assembly lines. CIRP Annals-Manufacturing Technology, 58(2):628-646, 2009.

103. Marek Kuczma. An introduction to the theory of functional equations and inequalities: Cauchy's equation and Jensen's inequality. Springer Science \& Business Media, 2009.

104. Alex Kuefler, Jeremy Morton, Tim A Wheeler, and Mykel J Kochenderfer. Imitating driver behavior with generative adversarial networks. In Proceedings of the IEEE Intelligent Vehicles Symposium (IV), 2017.

105. James J Kuffner and Steven M LaValle. RRT-connect: An efficient approach to singlequery path planning. In Proceedings of the IEEE International Conference on Robotics and Automation (ICRA), volume 2, pages 995-1001, 2000.

106. Joseph P La Salle. An invariance principle in the theory of stability. 1966.

107. Ioan Doré Landau, Rogelio Lozano, and Mohammed M'Saad. Adaptive control, volume 51. Springer, 1998.

108. Steven M LaValle and James J Kuffner Jr. Rapidly-exploring random trees: Progress and prospects. In Algorithmic and Computational Robotics: New Directions, pages 293-308, 2000.

109. Jessica Leber. At volkswagen, robots are coming out of their cages, 2013.

110. Yann LeCun, Yoshua Bengio, and Geoffrey Hinton. Deep learning. Nature, 521(7553):436, 2015. 
111. Insuk Lee, Eiru Kim, and Edward M Marcotte. Modes of interaction between individuals dominate the topologies of real world networks. PLOS ONE, 10(3):1-12, 2015.

112. Ian Lenz, Honglak Lee, and Ashutosh Saxena. Deep learning for detecting robotic grasps. The International Journal of Robotics Research, 34(4-5):705-724, 2015.

113. Sergey Levine, Chelsea Finn, Trevor Darrell, and Pieter Abbeel. End-to-end training of deep visuomotor policies. Journal of Machine Learning Research, 17(39):1-40, 2016.

114. Sergey Levine, Nolan Wagener, and Pieter Abbeel. Learning contact-rich manipulation skills with guided policy search. In Robotics and Automation (ICRA), 2015 IEEE International Conference on, pages 156-163. IEEE, 2015.

115. X Li, Z Sun, Z He, Q Zhu, and D Liu. A practical trajectory planning framework for autonomous ground vehicles driving in urban environments. In Proceedings of the IEEE Intelligent Vehicles Symposium (IV), pages 1160-1166, 2015.

116. Chu-Min Liao and Richard SW Masters. Analogy learning: A means to implicit motor learning. Journal of Sports Sciences, 19(5):307-319, 2001.

117. Hsien-Chung Lin, Changliu Liu, and Masayoshi Tomizuka. Fast robot motion planning with collision avoidance and temporal optimization. In 2018 International Conference on Control, Automation, Robotics and Vision (ICARCV), pages 29-35. IEEE, 2018.

118. Hsien-Chung Lin, Te Tang, Yongxiang Fan, and Masayoshi Tomizuka. A framework for robot grasp transferring with non-rigid transformation. In 2018 IEEE/RSJ International Conference on Intelligent Robots and Systems (IROS), pages 2941-2948, Oct 2018.

119. Hsien-Chung Lin, Te Tang, Yongxiang Fan, Yu Zhao, Masayoshi Tomizuka, and Wenjie Chen. Robot learning from human demonstration with remote lead through teaching. In Proceedings of the European Control Conference (ECC), 2016.

120. Hsien-Chung Lin, Te Tang, Masayoshi Tomizuka, and Wenjie Chen. Remote lead through teaching by human demonstration device. In Proceedings of the ASME Dynamic Systems and Control Conference (DSCC), pages V002T30A003V002T30A003, 2015.

121. Michael L Littman. Markov games as a framework for multi-agent reinforcement learning. In Proceedings of the International Conference on Machine Learning (ICML), volume 157, pages 157-163, 1994.

122. Chang Liu, S Lee, S Varnhagen, and HE Tseng. Path planning for autonomous vehicles using model predictive control. In Proceedings of the IEEE Intelligent Vehicles Symposium (IV), pages 174-179, 2017.

123. Changliu Liu. Real time robot motion planning in dynamic uncertain environment.

124. Changliu Liu, Jianyu Chen, Trong-Duy Nguyen, and Masayoshi Tomizuka. The robustly-safe automated driving system for enhanced active safety. In SAE Technical Paper, number 2017-01-1406, 2017.

125. Changliu Liu, Chung-Wei Lin, Shinichi Shiraishi, and Masayoshi Tomizuka. Distributed conflict resolution for connected autonomous vehicles. IEEE Transactions on Intelligent Vehicles, 3(1):1-12, 2018.

126. Changliu Liu, Chung-Yen Lin, and Masayoshi Tomizuka. The convex feasible set algorithm for real time optimization in motion planning. SIAM Journal on Control and Optimization, page in review, 2016.

127. Changliu Liu, Chung-Yen Lin, and Masayoshi Tomizuka. The convex feasible set algorithm for real time optimization in motion planning. SIAM Journal on Control and Optimization, 56(4):2712-2733, 2018. 
128. Changliu Liu, Chung-Yen Lin, Yizhou Wang, and Masayoshi Tomizuka. Convex feasible set algorithm for constrained trajectory smoothing. In Proceedings of the American Control Conference (ACC), pages 4177-4182, 2017.

129. Changliu Liu, Te Tang, Hsien-Chung Lin, Yujiao Cheng, and Masayoshi Tomizuka. Serocs: Safe and efficient robot collaborative systems for next generation intelligent industrial co-robots. arXiv preprint arXiv:1809.08215, 2018.

130. Changliu Liu and Masayoshi Tomizuka. Control in a safe set: Addressing safety in human robot interactions. In Proceedings of the ASME Dynamic Systems and Control Conference (DSCC), page V003T42A003, 2014.

131. Changliu Liu and Masayoshi Tomizuka. Modeling and controller design of cooperative robots in workspace sharing human-robot assembly teams. In Proceedings of the IEEE/RSJ International Conference on Intelligent Robots and Systems (IROS), pages 1386-1391, 2014.

132. Changliu Liu and Masayoshi Tomizuka. Safe exploration: Addressing various uncertainty levels in human robot interactions. In Proceedings of the American Control Conference (ACC), pages 465-470, 2015.

133. Changliu Liu and Masayoshi Tomizuka. Algorithmic safety measures for intelligent industrial co-robots. In Proceedings of the IEEE International Conference on Robotics and Automation (ICRA), pages 3095-3102, 2016.

134. Changliu Liu and Masayoshi Tomizuka. Enabling safe freeway driving for automated vehicles. In Proceedings of the American Control Conference (ACC), pages 34613467, 2016.

135. Changliu Liu and Masayoshi Tomizuka. Designing the robot behavior for safe human robot interactions. In Trends in Control and Decision-Making for Human-Robot Collaboration Systems, pages 241-270. Springer, 2017.

136. Changliu Liu and Masayoshi Tomizuka. Real time trajectory optimization for nonlinear robotic systems: Relaxation and convexification. Systems \& Control Letters, 108:5663, 2017.

137. Changliu Liu, Yizhou Wang, and Masayoshi Tomizuka. Boundary layer heuristic for search-based nonholonomic path planning in maze-like environments. In Proceedings of the IEEE Intelligent Vehicles Symposium (IV), pages 831-836, 2017.

138. Changliu Liu, Wei Zhan, and Masayoshi Tomizuka. Speed profile planning in dynamic environments via temporal optimization. In Proceedings of the IEEE Intelligent Vehicles Symposium (IV), pages 154-159, 2017.

139. Changliu Liu, Wenlong Zhang, and Masayoshi Tomizuka. Who to blame? Learning and control strategies with information asymmetry. In Proceedings of the American Control Conference (ACC), pages 4859-4864, 2016.

140. Jiming Liu. Autonomous Agents and Multi-Agent Systems: Explorations in Learning, Self-Organization, and Adaptive Computation. World Scientific, 2001.

141. Xinfu Liu. Autonomous trajectory planning by convex optimization. $\mathrm{PhD}$ thesis, Iowa State University, 2013.

142. Xinfu Liu and Ping Lu. Solving nonconvex optimal control problems by convex optimization. Journal of Guidance, Control, and Dynamics, 37(3):750-765, 2014.

143. Yun-Hui Liu. Qualitative test and force optimization of 3-d frictional form-closure grasps using linear programming. IEEE Transactions on Robotics and Automation, 15(1):163-173, 1999. 
144. Yun-Hui Liu. Computing n-finger form-closure grasps on polygonal objects. The International Journal of Robotics Research, 19(2):149-158, 2000.

145. Steve Lohr. The age of big data. New York Times, 11(2012), 2012.

146. Lu Lu and John T. Wen. Human-robot cooperative control for mobility impaired individuals. In Proceedings of the American Control Conference (ACC), pages 447-452, 2015.

147. Ren C Luo, Han B Huang, CY Yi, and Yi W Perng. Adaptive impedance control for safe robot manipulator. In Proceedings of the World Congress on Intelligent Control and Automation (WCICA), pages 1146-1151, 2011.

148. Ruikun Luo and Dmitry Berenson. A framework for unsupervised online human reaching motion recognition and early prediction. In Proceedings of the IEEE/RSJ International Conference on Intelligent Robots and Systems (IROS), pages 2426-2433, 2015.

149. Vadim Macagon and Burkhard Wünsche. Efficient collision detection for skeletally animated models in interactive environments. In Proceedings of Image and Vision Computing New Zealand (IVCNZ), volume 3, pages 378-383, 2003.

150. Jeffrey Mahler, Jacky Liang, Sherdil Niyaz, Michael Laskey, Richard Doan, Xinyu Liu, Juan Aparicio Ojea, and Ken Goldberg. Dex-net 2.0: Deep learning to plan robust grasps with synthetic point clouds and analytic grasp metrics. arXiv preprint arXiv:1703.09312, 2017.

151. Jeffrey Mahler, Florian T Pokorny, Brian Hou, Melrose Roderick, Michael Laskey, Mathieu Aubry, Kai Kohlhoff, Torsten Kröger, James Kuffner, and Ken Goldberg. Dexnet 1.0: A cloud-based network of $3 \mathrm{~d}$ objects for robust grasp planning using a multiarmed bandit model with correlated rewards. In Robotics and Automation (ICRA), 2016 IEEE International Conference on, pages 1957-1964. IEEE, 2016.

152. Jim Mainprice and Dmitry Berenson. Human-robot collaborative manipulation planning using early prediction of human motion. In Proceedings of the IEEE/RSJ International Conference on Intelligent Robots and Systems (IROS), pages 299-306, 2013.

153. Julie L Marble, David J Bruemmer, Douglas A Few, and Donald D Dudenhoeffer. Evaluation of supervisory vs. peer-peer interaction with human-robot teams. In Proceedings of the Annual Hawaii International Conference on System Sciences, pages $1-9,2004$.

154. Ian M Mitchell. Comparing forward and backward reachability as tools for safety analysis. In International Workshop on Hybrid Systems: Computation and Control, pages 428-443. Springer, 2007.

155. Volodymyr Mnih, Koray Kavukcuoglu, David Silver, Alex Graves, Ioannis Antonoglou, Daan Wierstra, and Martin Riedmiller. Playing atari with deep reinforcement learning. arXiv preprint arXiv:1312.5602, 2013.

156. Volodymyr Mnih, Koray Kavukcuoglu, David Silver, Andrei A Rusu, Joel Veness, Marc G Bellemare, Alex Graves, Martin Riedmiller, Andreas K Fidjeland, Georg Ostrovski, et al. Human-level control through deep reinforcemet learning. Nature, 518(7540):529-533, 2015.

157. Seyed Sajad Mousavi, Michael Schukat, and Enda Howley. Deep reinforcement learning: an overview. In Proceedings of SAI Intelligent Systems Conference, pages 426440. Springer, 2016.

158. Richard M Murray, Zexiang Li, S Shankar Sastry, and S Shankara Sastry. A mathematical Introduction to Robotic Manipulation. CRC Press, 1994.

159. Andriy Myronenko and Xubo Song. Point set registration: Coherent point drift. Pattern Analysis and Machine Intelligence, IEEE Transactions on, 32(12):2262-2275, 2010. 
160. Xiaoxiang Na and David J Cole. Linear quadratic game and non-cooperative predictive methods for potential application to modelling driver-AFS interactive steering control. Vehicle System Dynamics, 51(2):165-198, 2013.

161. Van-Duc Nguyen. Constructing force-closure grasps. The International Journal of Robotics Research, 7(3):3-16, 1988.

162. Stefanos Nikolaidis and Julie Shah. Human-robot cross-training: computational formulation, modeling and evaluation of a human team training strategy. In Proceedings of the ACM/IEEE International Conference on Human-Robot Interaction, pages 33-40, 2013.

163. Jorge Nocedal and Stephen Wright. Numerical optimization. Springer Science \& Business Media, Springer-Verlag New York, 2006.

164. Jeanne Ellis Ormrod and Kevin M Davis. Human learning. Merrill London, 2004.

165. Kartik Pandit, Dipak Ghosal, H Michael Zhang, and Chen-Nee Chuah. Adaptive traffic signal control with vehicular ad hoc networks. IEEE Transactions on Vehicular Technology, 62(4):1459-1471, 2013.

166. Chavdar Papazov, Sami Haddadin, Sven Parusel, Kai Krieger, and Darius Burschka. Rigid 3d geometry matching for grasping of known objects in cluttered scenes. The International Journal of Robotics Research, 31(4):538-553, 2012.

167. Raja Parasuraman, Thomas B Sheridan, and Christopher D Wickens. A model for types and levels of human interaction with automation. IEEE Transactions on Systems, Man, and Cybernetics-Part A: Systems and Humans, 30(3):286-297, 2000.

168. Dae-Hyung Park, Heiko Hoffmann, Peter Pastor, and Stefan Schaal. Movement reproduction and obstacle avoidance with dynamic movement primitives and potential fields. In Proceedings of the IEEE-RAS International Conference on Humanoid Robots, pages 91-98, 2008.

169. Hyun-Keun Park, Hyun Seok Hong, Han Jo Kwon, and Myung Jin Chung. A nursing robot system for the elderly and the disabled. International Journal of Human-Friendly Welfare Robotic Systems, 2(4):11-16, 2001.

170. Vladimir Pavlovic, James M Rehg, Tat-Jen Cham, and Kevin P Murphy. A dynamic Bayesian network approach to figure tracking using learned dynamic models. In Proceedings of the IEEE International Conference on Computer Vision (ICCV), volume 1, pages 94-101, 1999.

171. Leif E Peterson. K-nearest neighbor. Scholarpedia, 4(2):1883, 2009.

172. Lerrel Pinto and Abhinav Gupta. Supersizing self-supervision: Learning to grasp from 50k tries and 700 robot hours. In Robotics and Automation (ICRA), 2016 IEEE International Conference on, pages 3406-3413. IEEE, 2016.

173. Jean Ponce and Bernard Faverjon. On computing three-finger force-closure grasps of polygonal objects. IEEE Transactions on Robotics and Automation, 11(6):868-881, 1995.

174. Xiangjun Qian, Iñaki Navarro, Arnaud de La Fortelle, and Fabien Moutarde. Motion planning for urban autonomous driving using Bézier curves and MPC. In Proceedings of the IEEE International Conference on Intelligent Transportation Systems (ITSC), pages 826-833, 2016.

175. Eric Rasmusen and Basil Blackwell. Games and Information: An Introduction to Game Theory. Cambridge, MA, 1994.

176. Jens Rasmussen. Outlines of a hybrid model of the process plant operator. In Monitoring Behavior and Supervisory Control, pages 371-383. Springer, 1976. 
177. Nathan Ratliff, Matt Zucker, J Andrew Bagnell, and Siddhartha Srinivasa. CHOMP: Gradient optimization techniques for efficient motion planning. In Proceedings of the IEEE International Conference on Robotics and Automation (ICRA), pages 489-494, 2009.

178. Harish C Ravichandar and Ashwin Dani. Human intention inference and motion modeling using approximate e-m with online learning. In Proceedings of the IEEE/RSJ International Conference on Intelligent Robots and Systems (IROS), pages 1819-1824, 2015.

179. Harish C Ravichandar, Avnish Kumar, and Ashwin Dani. Bayesian human intention inference through multiple model filtering with gaze-based priors. In Proceedings of the International Conference on Information Fusion (FUSION), pages 2296-2302, 2016.

180. John H Reif and Hongyan Wang. Social potential fields: A distributed behavioral control for autonomous robots. Robotics and Autonomous Systems, 27(3):171 - 194, 1999.

181. Wei Ren, Randal W Beard, et al. Consensus seeking in multiagent systems under dynamically changing interaction topologies. IEEE Transactions on Automatic Control, 50(5):655-661, 2005.

182. GZ Rey, M Carvalho, and D Trentesaux. Cooperation models between humans and artificial self-organizing systems: Motivations, issues and perspectives. In Proceedings of the International Symposium on Resilient Control Systems (ISRCS), pages 156-161, 2013.

183. G Rigatos and S Tzafestas. Extended kalman filtering for fuzzy modelling and multi-sensor fusion. Mathematical and Computer Modelling of Dynamical Systems, 13(3):251-266, 2007.

184. Philip E Ross. California to issue driving licences to robots. IEEE Spectrum, 2014.

185. Dorsa Sadigh, Shankar Sastry, Sanjit A Seshia, and Anca D Dragan. Planning for autonomous cars that leverages effects on human actions. In Proceedings of the Robotics: Science and Systems Conference (RSS), 2016.

186. Andrey V Savkin, Alexey S Matveev, Michael Hoy, and Chao Wang. Safe Robot Navigation Among Moving and Steady Obstacles. Butterworth-Heinemann, 2015.

187. Stefan Schaal, Auke Ijspeert, and Aude Billard. Computational approaches to motor learning by imitation. Philosophical Transactions of the Royal Society B: Biological Sciences, 358(1431):537-547, 2003.

188. John Schulman, Yan Duan, Jonathan Ho, Alex Lee, Ibrahim Awwal, Henry Bradlow, Jia Pan, Sachin Patil, Ken Goldberg, and Pieter Abbeel. Motion planning with sequential convex optimization and convex collision checking. The International Journal of Robotics Research, 33(9):1251-1270, 2014.

189. John Schulman, Jonathan Ho, Alex X Lee, Ibrahim Awwal, Henry Bradlow, and Pieter Abbeel. Finding locally optimal, collision-free trajectories with sequential convex optimization. In Proceedings of the Robotics: Science and Systems Conference (RSS), volume 9, pages 1-10, 2013.

190. John Schulman, Jonathan Ho, Cameron Lee, and Pieter Abbeel. Learning from demonstrations through the use of non-rigid registration. In Proceedings of the 16th International Symposium on Robotics Research (ISRR), 2013.

191. Mac Schwager, Carrick Detweiler, Iuliu Vasilescu, Dean M Anderson, and Daniela Rus. Data-driven identification of group dynamics for motion prediction and control. Journal of Field Robotics, 25(6-7):305-324, 2008.

192. Loren Arthur Schwarz, Artashes Mkhitaryan, Diana Mateus, and Nassir Navab. Human skeleton tracking from depth data using geodesic distances and optical flow. Image and Vision Computing, 30(3):217-226, 2012. 
193. Elham Semsar-Kazerooni and Khashayar Khorasani. Multi-agent team cooperation: A game theory approach. Automatica, 45(10):2205 - 2213, 2009.

194. David Silver, Aja Huang, Chris J Maddison, Arthur Guez, Laurent Sifre, George Van Den Driessche, Julian Schrittwieser, Ioannis Antonoglou, Veda Panneershelvam, Marc Lanctot, et al. Mastering the game of go with deep neural networks and tree search. Nature, 529(7587):484-489, 2016.

195. Emrah A Sisbot, Luis Felipe Marin-Urias, Xavier Broquere, Daniel Sidobre, and Rachid Alami. Synthesizing robot motions adapted to human presence. International Journal of Social Robotics, 2(3):329-343, 2010.

196. S Skiena. Dijkstra's algorithm. Implementing Discrete Mathematics: Combinatorics and Graph Theory with Mathematica, Reading, MA: Addison-Wesley, pages 225-227, 1990.

197. Jean-Jacques E Slotine, Weiping Li, et al. Applied nonlinear control, volume 199. Prentice-Hall Englewood Cliffs, NJ, 1991.

198. Peter Spellucci. A new technique for inconsistent QP problems in the SQP method. Mathematical Methods of Operations Research, 47(3):355-400, 1998.

199. Roman G Strongin and Yaroslav D Sergeyev. Global optimization with non-convex constraints: Sequential and parallel algorithms, volume 45. Springer Science \& Business Media, 2013.

200. Liting Sun, Cheng Peng, Wei Zhan, and Masayoshi Tomizuka. A fast integrated planning and control framework for autonomous driving. In arXiv:1707.02515, 2017.

201. Wen Sun, Arun Venkatraman, Geoffrey J Gordon, Byron Boots, and J Andrew Bagnell. Deeply aggrevated: Differentiable imitation learning for sequential prediction. In Proceedings of the 34th International Conference on Machine Learning-Volume 70, pages 3309-3318. JMLR. org, 2017.

202. Supplementary website for Te Tang's dissertation. http://me.berkeley.edu/ $\sim$ tetang/Dissertation.

203. Susumu Tachi and Kiyoshi Komoriya. Guide dog robot. Autonomous Mobile Robots: Control, Planning, and Architecture, pages 360-367, 1984.

204. Tadele S Tadele, Theo JA de Vries, and Stefano Stramigioli. The safety of domestic robots: a survey of various safety-related publications. IEEE Robotics and Automation Magazine, pages 134-142, 2014.

205. JTC Tan, F Duan, Y Zhang, K Watanabe, R Kato, and T Arai. Human-robot collaboration in cellular manufacturing: Design and development. In Proceedings of the IEEE/RSJ International Conference on Intelligent Robots and Systems (IROS), pages 29-34, 2009.

206. Te Tang, Hsien-Chung Lin, and Masayoshi Tomizuka. A learning-based framework for robot peg-hole-insertion. In Proceedings of the ASME Dynamic Systems and Control Conference (DSCC), pages V002T27A002-V002T27A002, 2015.

207. Te Tang, Hsien-Chung Lin, Yu Zhao, Wenjie Chen, and Masayoshi Tomizuka. Autonomous alignment of peg and hole by force/torque measurement for robotic assembly. In Proceedings of the IEEE International Conference on Automation Science and Engineering (CASE), pages 162-167, 2016.

208. Te Tang, Hsien-Chung Lin, Yu Zhao, Yongxiang Fan, Wenjie Chen, and Masayoshi Tomizuka. Teach industrial robots peg-hole-insertion by human demonstration. In Proceedings of the IEEE International Conference on Advanced Intelligent Mechatronics (AIM), pages 488-494, 2016. 
209. Te Tang, Changliu Liu, Wenjie Chen, and Masayoshi Tomizuka. Robotic manipulation of deformable objects by tangent space mapping and non-rigid registration. In Proceedings of the IEEE/RSJ International Conference on Intelligent Robots and Systems (IROS), pages 2689-2696, 2016.

210. Mohit Tawarmalani and Nikolaos V Sahinidis. Convexification and Global Optimization in Continuous and Mixed-Integer Nonlinear Programming: Theory, Algorithms, Software, and Applications, volume 65. Springer Science \& Business Media, 2002.

211. Andrea Lockerd Thomaz, Cynthia Breazeal, et al. Reinforcement learning with human teachers: Evidence of feedback and guidance with implications for learning performance. In Proceedings of the National Conference on Artificial Intelligence (AAAI), volume 6, pages 1000-1005, 2006.

212. Sebastian Thrun and Tom M Mitchell. Lifelong robot learning. In The Biology and Technology of Intelligent Autonomous Agents, pages 165-196. Springer, 1995.

213. Kaoru Tone. Revisions of constraint approximations in the successive QP method for nonlinear programming problems. Mathematical Programming, 26(2):144-152, 1983.

214. Giovanni Tonietti, Riccardo Schiavi, and Antonio Bicchi. Design and control of a variable stiffness actuator for safe and fast physical human/robot interaction. In Proceedings of the IEEE International Conference on Robotics and Automation (ICRA), pages 526-531, 2005.

215. Peter Trautman and Andreas Krause. Unfreezing the robot: Navigation in dense, interacting crowds. In Proceedings of the IEEE/RSJ International Conference on Intelligent Robots and Systems (IROS), pages 797-803, 2010.

216. Chi-Shen Tsai, Jwu-Sheng Hu, and Masayoshi Tomizuka. Ensuring safety in humanrobot coexistence environment. In Proceedings of the IEEE/RSJ International Conference on Intelligent Robots and Systems (IROS), pages 4191-4196, 2014.

217. Annemarie Turnwald, Wiktor Olszowy, Dirk Wollherr, and Martin Buss. Interactive navigation of humans from a game theoretic perspective. In Proceedings of the IEEE/RSJ International Conference on Intelligent Robots and Systems (IROS), pages 703-708, 2014.

218. Gündüz Ulusoy, Funda Sivrikaya-Şerifoğlu, and Ümit Bilge. A genetic algorithm approach to the simultaneous scheduling of machines and automated guided vehicles. Computers and Operations Research, 24(4):335-351, 1997.

219. Chris Urmson, Joshua Anhalt, Drew Bagnell, Christopher Baker, Robert Bittner, MN Clark, John Dolan, Dave Duggins, Tugrul Galatali, Chris Geyer, et al. Autonomous driving in urban environments: Boss and the urban challenge. Journal of Field Robotics, 25(8):425-466, 2008.

220. Jur van den Berg. Extended LQR: locally-optimal feedback control for systems with non-linear dynamics and non-quadratic cost. In Robotics Research, pages 39-56. Springer, 2016.

221. Jur Van Den Berg, Pieter Abbeel, and Ken Goldberg. LQG-MP: Optimized path planning for robots with motion uncertainty and imperfect state information. The International Journal of Robotics Research, 30(7):895-913, 2011.

222. Timothy Van Zandt. Interim bayesian nash equilibrium on universal type spaces for supermodular games. Journal of Economic Theory, 145(1):249-263, 2010.

223. Jacob Varley, Jonathan Weisz, Jared Weiss, and Peter Allen. Generating multi-fingered robotic grasps via deep learning. In Intelligent Robots and Systems (IROS), 2015 IEEE/RSJ International Conference on, pages 4415-4420. IEEE, 2015. 
224. Andreas Vlachos. An investigation of imitation learning algorithms for structured prediction. In European Workshop on Reinforcement Learning, pages 143-154, 2013.

225. Michael Wooldridge. An Introduction to Multiagent Systems. John Wiley \& Sons, 2009.

226. Ping Wu, Yang Cao, Yuqing He, and Decai Li. Vision-based robot path planning with deep learning. In International Conference on Computer Vision Systems, pages 101111. Springer, 2017.

227. Wenda Xu, Junqing Wei, J.M. Dolan, Huijing Zhao, and Hongbin Zha. A real-time motion planner with trajectory optimization for autonomous vehicles. In Proceedings of the IEEE International Conference on Robotics and Automation (ICRA), pages 2061-2067, 2012.

228. Chenguang Yang, Gowrishankar Ganesh, Sami Haddadin, Sven Parusel, Alin AlbuSchaeffer, and Etienne Burdet. Human-like adaptation of force and impedance in stable and unstable interactions. IEEE Transactions on Robotics, 27(5):918-930, 2011.

229. Shichao Yang, Sandeep Konam, Chen Ma, Stephanie Rosenthal, Manuela Veloso, and Sebastian Scherer. Obstacle avoidance through deep networks based intermediate perception. arXiv preprint arXiv:1704.08759, 2017.

230. James E Young, Richard Hawkins, Ehud Sharlin, and Takeo Igarashi. Toward acceptable domestic robots: Applying insights from social psychology. International Journal of Social Robotics, 1(1):95-108, 2009.

231. Wenlong Zhang, Xu Chen, Joonbum Bae, and Masayoshi Tomizuka. Real-time kinematic modeling and prediction of human joint motion in a networked rehabilitation system. In American Control Conference (ACC), pages 5800-5805, 2015.

232. Yu Zhang, Huiyan Chen, Steven L Waslander, Tian Yang, Sheng Zhang, Guangming Xiong, and Kai Liu. Speed planning for autonomous driving via convex optimization. In 2018 21st International Conference on Intelligent Transportation Systems (ITSC), pages 1089-1094. IEEE, 2018.

233. Zhenyue Zhang and Jing Wang. Mlle: Modified locally linear embedding using multiple weights. In Advances in Neural Information Processing Systems, pages 1593-1600, 2007.

234. Zhijie Zhu, Edward Schmerling, and Marco Pavone. A convex optimization approach to smooth trajectories for motion planning with car-like robots. In Proceedings of the IEEE Conference on Decision and Control (CDC), pages 835-842, 2015.

235. Michael Zinn, Bernard Roth, Oussama Khatib, and J Kenneth Salisbury. A new actuation approach for human friendly robot design. The International Journal of Robotics Research, 23(4-5):379-398, 2004. 